FR-PHENO-2011-002

BONN-TH-2011-02

DESY 11-016

\title{
HiggsBounds 2.0.0: Confronting Neutral and Charged Higgs Sector Predictions with Exclusion Bounds from LEP and the Tevatron
}

\author{
P. Bechtle ${ }^{a}$, O. Brein ${ }^{b}$, S. Heinemeyer ${ }^{c}$, G. Weiglein ${ }^{a}$ \\ and K.E. Williams ${ }^{\mathrm{d}}$. \\ a DESY, Notkestrasse 85, 22607 Hamburg, Germany \\ b Physikalisches Institut, Albert-Ludwigs-Universität Freiburg, \\ Hermann-Herder-Str. 3, D-79104 Freiburg im Breisgau, Germany \\ ${ }^{\mathrm{c}}$ Instituto de Física de Cantabria (CSIC-UC), Santander, Spain \\ d Bethe Center for Theoretical Physics, Physikalisches Institut der Universität Bonn, \\ Nussallee 12, 53115 Bonn, Germany
}

\begin{abstract}
HiggsBounds 2.0.0 is a computer code which tests both neutral and charged Higgs sectors of arbitrary models against the current exclusion bounds from the Higgs searches at LEP and the Tevatron. As input, it requires a selection of model predictions, such as Higgs masses, branching ratios, effective couplings and total decay widths. HiggsBounds 2.0.0 then uses the expected and observed topological cross section limits from the Higgs searches to determine whether a given parameter scenario of a model is excluded at the 95\% C.L. by those searches. Version 2.0.0 represents a significant extension of the code since its first release (1.0.0). It includes now 28/53 LEP/Tevatron Higgs search analyses, compared to the $11 / 22$ in the first release, of which many of the ones from the Tevatron are replaced by updates. As a major extension, the code allows now the predictions for (singly) charged Higgs bosons to be confronted with LEP and Tevatron searches. Furthermore, the newly included analyses contain LEP searches for neutral Higgs bosons $(H)$ decaying invisibly or into (non flavour tagged) hadrons as well as decay-mode independent searches for neutral Higgs bosons, LEP searches via the production modes $\tau^{+} \tau^{-} H$ and $b \bar{b} H$, and Tevatron searches via $t \bar{t} H$. Also, all Tevatron results presented at the ICHEP'10 are included in version 2.0.0. As physics applications of HiggsBounds 2.0.0 we study the allowed Higgs mass range for model scenarios with invisible Higgs decays and we obtain exclusion results for the scalar sector of the Randall-Sundrum model using up-to-date LEP and Tevatron direct search results.
\end{abstract}




\section{Introduction}

The search for Higgs bosons is a major cornerstone of the physics programmes of past, present and future high energy colliders. The LEP and Tevatron experiments, in particular, have been able to turn the non-observation of Higgs bosons into constraints on the Higgs sector, which can be very useful in restricting the available parameter space of particle physics models. At the LHC, discoveries involving colour charged particles (e.g. squarks, gluinos) may occur already before a Higgs signal gets established. The first LHC Higgs search results from ATLAS and CMS are expected to become public during the next months. It is important to note that limits from the Higgs searches at the LHC, the Tevatron and LEP will play a crucial role also for testing the validity of model interpretations of possible Higgs-like signals or other signals of new physics.

The Higgs searches performed by the experimental collaborations usually take one of two forms. Dedicated analyses have been carried out in order to constrain some of the most popular models, such as the Standard Model (SM) [1] and various benchmark scenarios in the Minimal Supersymmetric Standard Model (MSSM) [2]. In addition, (essentially) model-independent limits on the cross sections of individual signal topologies (such as $e^{+} e^{-} \rightarrow h_{i} Z \rightarrow b \bar{b} Z$ ) have been published. The former type of analyses include detailed knowledge of the overlap between the individual experimental searches, and therefore have a high sensitivity, whereas the latter can be used to test a wide class of models.

There are certain issues involved with the application of these experimental constraints. The data is distributed over many different publications and the limits are given with a variety of normalisations. In the case of the Tevatron, the results are also frequently updated. Furthermore, care must be taken when using more than one experimental analysis to ensure the correct statistical interpretation of the result in terms of a confidence level (C.L.). One needs to take into account the underlying assumptions of the various experimental analyses in order to determine which analysis is applicable to which Higgs boson of a particular model. For example, if an analysis has been carried out assuming the validity of the SM, it will not be applicable to Higgs bosons whose various production and decay mechanisms do not show a proportionate similarity to the SM.

The Fortran code HiggsBounds has been designed to facilitate the task of comparing Higgs sector predictions with existing exclusion limits, thus allowing the user to quickly and conveniently check a wide variety of models against the state-of-the-art results from Higgs searches. HiggsBounds 2.0.0 represents a significant extension of the code since its first release in February 2009 [3] and its latest release in December 2009 (version 1.2.0). As a major extension, it allows now the predictions of (singly) charged Higgs sectors to be compared against current experimental limits from the LEP and Tevatron experiments. We have also significantly extended the 
variety of neutral Higgs searches included. From the LEP experiments, we newly included searches where the Higgs boson is assumed to decay invisibly or into $\gamma \gamma$ or (non flavour tagged) hadrons. Also newly included are the decay-mode independent search of OPAL [4] and several searches involving the production process $e^{+} e^{-} \rightarrow f \bar{f} H$ with $f \in\{b, \tau\}$. From the Tevatron experiments, we newly included the analyses released publicly at the time of the ICHEP 2010 Conference, thus replacing many implemented analyses by their updated versions. Furthermore, we newly include the CDF and D0 combination of their searches for the individual signal topology exclusive neutral Higgs boson production with decay into $W^{+} W^{-}[5]$, which is applicable to a wide class of models beyond the SM, a search via the $t \bar{t} H$ production process of [6] and a search assuming the decay $H \rightarrow \gamma Z$ [7]. A full list of the experimental analyses and the respective references included in HiggsBounds 2.0.0 is given in Sect. 4.

In this release, we do not yet include charged Higgs search channels which involve the decay of charged Higgs bosons to neutral ones plus a $W$ boson $H_{i}^{ \pm} \rightarrow h_{j}^{0} W^{ \pm}$. Neither are search results for doubly charged Higgs bosons included. Therefore, we will refer to the singly charged Higgs bosons below just as charged Higgs bosons.

The rest of the paper is organised as follows. In Section 2, we give a brief description of the general approach of the program HiggsBounds2.0.0. Section 3 describes the model predictions required by the program as input in order to enable the application of Higgs search results. The three input options of HiggsBounds are described in detail, illustrating the input required from the user in each case and indicating how this input is further processed. Section 4 lists all analyses and their corresponding references implemented in version 2.0.0 of the program. Furthermore, the conditions implemented in the code which are responsible for determining the applicability of some analyses to certain Higgs bosons are described here. The complete operating instructions of HiggsBounds2.0.0 for the library of subroutines, the command-line and the online version with examples are given in Section 5. In Section 6, we present two new applications of the program. One application considers a SM-like Higgs boson where an extra Higgs decay channel to invisible particles is open; the other studies the scalar sector of the Randall-Sundrum model and shows, to our knowledge, for the first time the impact of Tevatron Higgs search results on this model.

\section{General Approach}

The following is a short, but self contained, description of the basic work-flow of HiggsBounds. A more detailed treatment, e.g. of the limitations of the methods employed here, can be found in the manual of HiggsBounds 1.0.0 [3].

The user provides the Higgs sector predictions of the model under consideration. For each neutral or charged Higgs boson $H_{i}\left(i=1, \ldots, n_{H^{0}}+n_{H^{ \pm}}\right)$in the model, this includes information on the Higgs mass, Higgs total decay width, Higgs and top quark 
branching ratios and Higgs production cross sections,

$$
M_{H_{i}}, \Gamma_{\text {tot }}\left(H_{i}\right), \mathrm{BR}_{\text {model }}\left(H_{i} \rightarrow \ldots\right), \mathrm{BR}_{\text {model }}(t \rightarrow \ldots), \frac{\sigma_{\text {model }}(P)}{\sigma_{\text {ref }}(P)},
$$

and information whether each neutral Higgs boson is CP-odd, CP-even or is not a CP eigenstate. $P$ stands for the Higgs production process. A full list of the required Higgs sector predictions is given in Sect. 3.1. Where it exists, $\sigma^{\mathrm{SM}}(P)$ is used as the reference production cross section. Variations on the input format (1) are offered, as described in detail in Sect. 3, which allow the user to specify a simpler set of input, provided certain basic approximations are valid.

Note that the user may, to some extent, use the program also with incomplete model information by setting not provided cross section ratios, branching ratios or effective couplings to zero. However, the program may then consider some analyses not applicable, which would be taken into account if more complete model information had been provided.

The HiggsBounds package includes sample files which demonstrate how the code can be used in conjunction with the widely used programs for predictions in the MSSM Higgs sector FeynHiggs [8-11] and CPsuperH [12,13]. The possibility to link HiggsBounds with the codes DarkSUSY [14], Fittino [15], MasterCode [16,17], 2HDMC [18] has already been demonstrated in various applications.

A list of the experimental analyses currently included in HiggsBounds 2.0.0 can be generated by running the code. These include results from both LEP and the Tevatron and consist of tables of expected cross section limits (based on Monte Carlo simulations with no signal) and observed ones at 95\% C.L., with a variety of normalisations. The list mainly consists of analyses for which model-independent limits were published. However, we also include some dedicated analyses carried out for the case of the SM, or for Higgs bosons with certain CP properties. These analyses can only be applied to Higgs bosons which show the corresponding characteristics to a sufficiently high level of accuracy.

We call the application of a Higgs search analysis to a particular Higgs boson (or two Higgs bosons, if the analysis involves two of them) of the model under study with particular mass(es) an "analysis application" $X .^{1}$ To each analysis application $X$ corresponds a signal cross section prediction $\sigma(X)$ for the particular Higgs boson on which an upper limit is put by the analysis. For each $X$, HiggsBounds uses the input to calculate the relevant quantity $Q_{\text {model }}(X)$ in terms of which the limit is given (i.e., a conveniently normalised cross section $\sigma(X)$ times branching ratio).

1 As an example, suppose a model with three neutral Higgs bosons $\left(h_{1}, h_{2}, h_{3}\right)$ should be checked against two neutral Higgs search analyses $A_{1}$ and $A_{2}$, then there are six possible analysis applications $X\left(A_{1}\right.$ applied to $h_{1}, h_{2}, h_{3}$ and $A_{2}$ applied to $\left.h_{1}, h_{2}, h_{3}\right)$ that the model can be tested against. 
The normalisation is carried out using SM predictions for Higgs boson production cross sections and decay branching ratios from HDECAY [19] version 3.4, the TEV4LHC Higgs Working Group [20] (for a full list of references, see Tab. 11), VFB@NLO [21], HJET [22-24] version 1.3 and dedicated calculations of our own [3].

The rationale behind the choice of SM normalisation is that virtually all Tevatron analyses implemented in this program use these predictions when normalising their cross section limits to SM quantities. Thus, describing deviations from the SM of a new model by using the SM normalisation the experimental analyses have chosen allows for the most accurate interpretation of the limits. By using SM predictions for Higgs production cross sections which deviate from the ones used in the experimental analyses by a certain amount (be it because of a different loop order, different numerical values of input parameters, renormalisation scheme or choice of parton distribution functions), a deviation of the same relative size will be caused in the quantities $Q_{\text {model }}$. The user should bear this in mind when interpreting the output of HiggsBounds.

In order to ensure the correct statistical interpretation of the results, it is crucial to only consider the experimentally observed limit for one particular analysis application. Therefore, HiggsBounds must first determine $X_{0}$, which is defined as the analysis application with the highest statistical sensitivity for the model point under consideration. In order to do this, the program uses the tables of expected experimental limits to obtain a quantity $Q_{\text {expec }}$ corresponding to each $X$. The analysis application with the largest value of $Q_{\text {model }} / Q_{\text {expec }}$ is chosen as $X_{0}$.

HiggsBounds then determines a value for $Q_{\text {obs }}$ for this process $X_{0}$, using the appropriate table of experimentally observed limits. If

$$
\frac{Q_{\text {model }}\left(X_{0}\right)}{Q_{\text {obs }}\left(X_{0}\right)}>1
$$

HiggsBounds returns as a result that this particular parameter point is excluded at $95 \%$ C.L. ${ }^{2}$

In order to use HiggsBounds, the narrow-width approximation must be valid for each Higgs boson described in the input, because the experimental exclusion bounds currently utilised in the program have all been obtained under this assumption. HiggsBounds can be used with models which do not change the signature of the background processes considerably ${ }^{3}$. In addition, models should not significantly change

$\overline{2}$ Note that if we had compared predicted cross sections directly against the experimentally measured limits for all available search channels and had excluded the model if at least one of them excludes it at $95 \%$ C.L., the derived constraint would in general not correspond to a constraint at $95 \%$ C.L. The maximally $5 \%$ probability of each individual comparison of $Q_{\text {model }}$ and $Q_{\text {obs }}$ to yield a false exclusion would yield an overall probability for false exclusion which is higher than $5 \%$.

3 However, new physics models which show strong deviations from the SM in the back- 
the kinematical distributions of the signal cross section associated with $X$ (e.g. $\eta$, $p_{T}$ distributions of the final state particles) from that assumed in the corresponding analysis (for a full discussion see [3]).

In addition, the LEP Higgs vector boson fusion (VBF) cross section should not be significantly enhanced compared to the LEP Higgsstrahlung cross section. (Models with a common $g_{h_{i} V V} / g_{H V V}^{\mathrm{SM}}$ coupling for $V=Z, W$ and no new mediating particles, such as the MSSM, satisfy this condition automatically.)

\section{Theoretical predictions required as input}

The user can choose between three different input formats, labelled by the variable whichinput. We describe here in detail what is required from the user for each of the three settings (hadr, part and effC). A summary can be found in Tab. 3, Sect. 5.1.

\subsection{The most general input form: whichinput=hadr}

The option whichinput=hadr requires the model input in the most general form. It involves specifying (at most)

(1) masses for the neutral Higgs bosons $h_{k}\left(k=1, n_{H^{0}}\right)$ and singly charged Higgs bosons $H_{j}^{ \pm}\left(j=1, n_{H^{ \pm}}\right)$,

$$
m_{h_{k}}, m_{H_{j}^{ \pm}}
$$

(2) Higgs total decay widths,

$$
\Gamma_{\text {tot }}\left(h_{k}\right), \Gamma_{\text {tot }}\left(H_{k}^{ \pm}\right)
$$

(3) whether the neutral Higgs boson is CP-even, CP-odd or mixed CP,

(4) neutral Higgs branching ratios with $\mathrm{SM}$ equivalents ( $\mathrm{OP}=$ ordinary particles i.e. particles which exist in the SM),

$$
\mathrm{BR}_{\text {model }}\left(h_{k} \rightarrow \mathrm{OP}\right) \text { with } \mathrm{OP}=s \bar{s}, c \bar{c}, b \bar{b}, \tau^{+} \tau^{-}, W^{+} W^{-}, Z Z, Z \gamma, \gamma \gamma, g g
$$

(5) neutral Higgs branching ratios without SM equivalents

$$
\mathrm{BR}_{\text {model }}\left(h_{k} \rightarrow h_{i} h_{i}\right), \mathrm{BR}_{\text {model }}\left(h_{k} \rightarrow \text { invisible }\right),
$$

ground processes to Higgs production are not usually considered in the literature, since this would often put them in conflict with SM electroweak precision data [25,26]. 
(6) charged Higgs branching ratios to SM particles

$$
\mathrm{BR}\left(H_{j}^{+} \rightarrow \mathrm{OP}\right) \text { with } \mathrm{OP}=c \bar{s}, c \bar{b}, \tau^{+} \nu_{\tau},
$$

(7) top quark branching ratios

$$
\mathrm{BR}\left(t \rightarrow W^{+} b\right), \mathrm{BR}\left(t \rightarrow H_{j}^{+} b\right),
$$

(8) normalised cross section $R_{\sigma}(P)$ for LEP Higgs production process $P$

$$
\begin{aligned}
& e^{+} e^{-} \rightarrow h_{j} Z, \\
& e^{+} e^{-} \rightarrow b \bar{b} h_{j}, \\
& e^{+} e^{-} \rightarrow \tau^{+} \tau^{-} h_{j}, \\
& e^{+} e^{-} \rightarrow h_{j} h_{i}, \\
& e^{+} e^{-} \rightarrow H_{j}^{+} H_{j}^{-},
\end{aligned}
$$

(9) normalised cross section $R_{\sigma}(P)$ for Tevatron hadronic Higgs production process $P$

$$
\begin{aligned}
& p \bar{p} \rightarrow h_{j}, \\
& p \bar{p} \rightarrow b h_{j}, \\
& p \bar{p} \rightarrow h_{j} W, \\
& p \bar{p} \rightarrow h_{j} Z, \\
& p \bar{p} \rightarrow h_{j} \text { via VBF, } \\
& p \bar{p} \rightarrow t \bar{t} h_{j} .
\end{aligned}
$$

Note that only a subset of these inputs may be required. For example, if only the LEP limits are required by the user, no Tevatron cross sections will need to be given as input (see Sect. 5). If the user only wishes to test the neutral Higgs sector, no input involving the charged Higgs sector or top decays will be required.

The normalised cross section of a Higgs production process $P$ is defined by

$$
R_{\sigma}(P)=\frac{\sigma_{\text {model }}(P)}{\sigma_{\text {ref }}(P)}
$$

Where the SM equivalent exists, the reference cross section $\sigma_{\text {ref }}(P)$ of a process $P$ involving a Higgs boson $h_{k}$ is $\sigma_{\text {ref }}(P)=\left.\sigma_{\mathrm{SM}}(P)\right|_{m_{H}^{\mathrm{SM}}=m_{h_{k}}}$.

The only neutral Higgs production process without a SM equivalent considered in HiggsBounds up to now is $P=e^{+} e^{-} \rightarrow h_{j} h_{i}$. In this case, as in HiggsBounds 1.0.0 [2], we choose a reference cross section for a fictitious production process of two scalar particles $\left(H^{\prime}, H\right)$ with masses $m_{H^{\prime}}=m_{h_{j}}$ and $m_{H}=m_{h_{i}}$ via a virtual $Z$ exchange 
with a standardised squared coupling constant:

$$
\left(g_{H^{\prime} H Z}^{\mathrm{ref}}\right)^{2}=\frac{e^{2}}{4 s_{\mathrm{w}}^{2} c_{\mathrm{w}}^{2}},
$$

where $e$ denotes the electromagnetic coupling constant, and $s_{\mathrm{w}}$ and $c_{\mathrm{w}}$ the sine and cosine of the weak mixing angle, respectively.

The cross section of this process in leading order is then completely determined by the Higgs masses and SM input and related to the SM Higgsstrahlung cross section via a simple phase space factor:

$$
\begin{aligned}
\sigma_{\text {ref }}\left(H^{\prime} H \text { production }\right) & =\bar{\lambda}\left(m_{H^{\prime}}, m_{H}, s\right) \sigma_{H Z}^{\mathrm{SM}}\left(m_{H}\right), \\
\bar{\lambda}\left(m_{H^{\prime}}, m_{H}, s\right) & =\frac{\lambda_{H^{\prime} H}^{3 / 2}(s)}{\lambda_{H Z}^{1 / 2}(s)\left(\lambda_{H Z}(s)+12 \frac{m_{Z}^{2}}{s}\right)}, \\
\lambda_{a b}(s) & =\left[1-\frac{\left(m_{a}+m_{b}\right)^{2}}{s}\right]\left[1-\frac{\left(m_{a}-m_{b}\right)^{2}}{s}\right] .
\end{aligned}
$$

This reference cross section coincides with the MSSM tree level cross section for the process $e^{+} e^{-} \rightarrow h^{0} A^{0}$ if the Higgs mixing-angle dependent factor $\cos (\beta-\alpha)$ is divided out of the tree level coupling and $m_{A^{0}}$ and $m_{h^{0}}$ are chosen as $m_{H^{\prime}}$ and $m_{H}$. Therefore, $R_{\sigma}\left(e^{+} e^{-} \rightarrow h^{0} A^{0}\right)$ is simply given by $\cos ^{2}(\beta-\alpha)$ in the MSSM (with real parameters) at tree level.

For the process $P=e^{+} e^{-} \rightarrow H_{j}^{+} H_{j}^{-}$, the reference cross section is the cross section of the process $e^{+} e^{-} \rightarrow H^{+} H^{-}$in a 2 Higgs doublet model (e.g. the MSSM) at tree-level (i.e. s-channel $\gamma$ and $Z$ exchange). This reference cross section depends solely on the mass of the charged Higgs boson and SM quantities. As a consequence, in the MSSM, $R_{\sigma}\left(e^{+} e^{-} \rightarrow H_{j}^{+} H_{j}^{-}\right)=1$.

The invisible branching ratio $\mathrm{BR}_{\text {model }}\left(h_{k} \rightarrow\right.$ invisible $)$ is defined as the branching ratio of a neutral Higgs boson into particles which only appear in the detector as missing energy. Examples of invisible particles include neutralinos in the MSSM or majorons in supersymmetric models with spontaneous breaking of R-parity.

The top quark branching ratios $\mathrm{BR}\left(t \rightarrow H_{j}^{+} b\right)$ and $\mathrm{BR}\left(t \rightarrow W^{+} b\right)$ are also required as input, when the model is compared with the charged Higgs searches from the Tevatron. $\mathrm{BR}\left(t \rightarrow W^{+} b\right)$ is required for analyses in order to determine when analyses which assume $\mathrm{BR}\left(t \rightarrow H_{j}^{+} b\right)+\mathrm{BR}\left(t \rightarrow W^{+} b\right) \sim 1$ can be applied to the model point.

In order to make it more convenient for users to calculate this input, the HiggsBounds library provides a series of Fortran functions which allow the user to access the predictions of certain SM quantities, including the SM Tevatron hadronic Higgs production cross sections, total decay width and branching ratios as a function of Higgs mass and $\Gamma^{\mathrm{SM}}\left(t \rightarrow W^{+} b\right)$ as function of the top mass. These are listed in Tab. 11 together 
with the corresponding references.

\subsection{Specifying some cross sections at the partonic level: whichinput=part}

The input required for the option whichinput=part may be more convenient for the user to calculate than for the option whichinput=hadr, since most of the Tevatron cross section ratios can be specified at the partonic level rather than the hadronic level.

The input option whichinput=part requires, at most,

(1)-(8) as for whichinput=hadr

(9) normalised cross section $R_{\sigma}(P)$ for Tevatron hadronic neutral Higgs production process $P$

$$
\begin{aligned}
& p \bar{p} \rightarrow h_{j} \text { via } \mathrm{VBF} \\
& p \bar{p} \rightarrow t \bar{t} h_{j}
\end{aligned}
$$

(10) normalised cross section $R_{n m}^{h_{j}+y}$ for Tevatron partonic neutral Higgs production process $n m \rightarrow h_{j}+y$, where $y$ indicates a particle produced in association with the Higgs boson (or none if $y$ is omitted):

$$
\begin{aligned}
R_{n m}^{h_{j}}, \text { with } n m & =g g, b \bar{b}, \\
R_{n m}^{h_{j}+W^{+}}, \text {with } n m & =u \bar{d}, c \bar{s}, \\
R_{n m}^{h_{j}+W^{-}}, \text {with } n m & =d \bar{u}, s \bar{c}, \\
R_{n m}^{h_{j}+Z}, \text { with } n m & =d \bar{d}, u \bar{u}, s \bar{s}, c \bar{c}, b \bar{b}, \\
R_{n m}^{h_{j}+b, h_{j}+\bar{b}}, \text { with } n m & =b g, \bar{b} g
\end{aligned}
$$

The normalised cross section $R_{n m}^{h_{j}+y}$ for a Tevatron partonic neutral Higgs production process is defined by

$$
R_{n m}^{H+y}=\frac{\hat{\sigma}_{n m \rightarrow H+y}^{\text {model }}}{\hat{\sigma}_{n m \rightarrow H+y}^{\mathrm{SM}}}
$$

calculated for a parton-system centre-of-mass energy squared $\hat{s}=\hat{s}_{0}$, where $\hat{s}_{0}$ denotes the partonic production threshold $\hat{s}_{0}=\left(m_{H}+m_{y}\right)^{2}$ (with $m_{y}=0$ in the case of single Higgs boson production). This requires that the dependence on $\hat{s}$ is (at most) mild.

Internally, HiggsBounds uses the relation

$$
R_{\sigma}(P) \approx \sum_{\{n, m\}} R_{n m}^{H+y} \frac{\sigma_{\mathrm{SM}}(p \bar{p} \rightarrow n m \rightarrow H+y)}{\sigma_{\mathrm{SM}}(p \bar{p} \rightarrow H+y)}
$$


to calculate the hadronic cross section ratios from the partonic cross section ratios. The ratios $\frac{\sigma_{\mathrm{SM}}(p \bar{p} \rightarrow n m \rightarrow H+y)}{\sigma_{\mathrm{SM}}(p \bar{p} \rightarrow H+y)}$ are provided within HiggsBounds. (For further explanation of the applicability of this approximation and details of how $\frac{\sigma_{\mathrm{SM}}(p \bar{p} \rightarrow n m \rightarrow H+y)}{\sigma_{\mathrm{SM}}(p \bar{p} \rightarrow H+y)}$ are calculated, see [3]).

The partonic cross section ratios $R_{n m}^{H+y}$ are much easier to calculate than hadronic cross section ratios in a wide range of models. In addition, it is often possible to make the approximation

$$
R_{n m}^{h_{j}+W^{+}}=R_{n m}^{h_{j}+W^{-}}=R_{n m}^{h_{j}+Z}=: R^{h_{j}+V}
$$

for all $\mathrm{nm}$, thus substantially reducing the number of partonic cross section ratios which need to be provided by the user from twelve to four.

For instance in the MSSM with real parameters, this ratio $R^{h_{j}+V}$ can be calculated approximately from the normalised squared effective Higgs coupling to two Z bosons, i.e. $\left(g_{h_{j} Z Z}^{\text {model }} / g_{H Z Z}^{\mathrm{SM}}\right)^{2}$ (for definition of this coupling, see Sect. 3.3). Similarly, $R_{b \bar{b}}^{h_{j}}$ and $R_{b g, \bar{b} g}^{h_{j}+b, h_{j}+\bar{b}}$ can be taken to be approximately equal in the MSSM (and can also be calculated approximately from effective couplings: see Eq. (23)).

\subsection{The effective coupling approximation: whichinput=effC}

The option whichinput=effC allows the user to specify a greatly reduced number of input parameters. It involves specifying (at most)

(1) masses for the neutral Higgs bosons $h_{k}\left(k=1, n_{H^{0}}\right)$ and singly charged Higgs bosons $H_{j}^{ \pm}\left(j=1, n_{H^{ \pm}}\right)$,

$$
m_{h_{k}}, m_{H_{j}^{ \pm}}
$$

(2) Higgs total decay widths,

$$
\Gamma_{\text {tot }}\left(h_{k}\right), \Gamma_{\text {tot }}\left(H_{k}^{ \pm}\right)
$$

(3) normalised squared scalar and pseudoscalar effective couplings to fermions

$$
\left(\frac{g_{s, h_{k}(\mathrm{OP})}^{\text {model }}}{g_{H(\mathrm{OP})}^{\mathrm{SM}}}\right)^{2},\left(\frac{g_{p, h_{k}(\mathrm{OP})}^{\text {model }}}{g_{H(\mathrm{OP})}^{\mathrm{SM}}}\right)^{2}, \mathrm{OP}=s \bar{s}, c \bar{c}, b \bar{b}, t \bar{t}, \tau^{+} \tau^{-}
$$

(4) normalised squared effective couplings to bosons

$$
\left(\frac{g_{h_{i} h_{j} Z}^{\text {model }}}{g_{H^{\prime} H Z}^{\text {ref }}}\right)^{2},\left(\frac{g_{h_{k}(\mathrm{OP})}^{\text {model }}}{g_{H(\mathrm{OP})}^{\mathrm{SM}}}\right)^{2}, \mathrm{OP}=W^{+} W^{-}, Z Z, Z \gamma, \gamma \gamma, g g
$$


(5) neutral Higgs branching ratios without SM equivalents, charged Higgs branching ratios to SM particles and top quark branching ratios as before

From this input, the input corresponding to the option part is calculated.

We define the scalar and pseudoscalar Higgs coupling to fermions in the usual way, via the Feynman rule for the coupling of a generic neutral Higgs boson $h$ to fermions:

$$
G(h f \bar{f})=i\left(g_{s} \mathbf{1}+i g_{p} \gamma_{5}\right)
$$

where $g_{s}$ and $g_{p}$ are real-valued scalar and pseudoscalar coupling constants respectively, and 1 and $\gamma_{5}$ are the usual matrices in Dirac space. A scalar particle, like the SM Higgs boson, has $g_{p}=0$ and a pseudoscalar particle has $g_{s}=0$.

Where it exists, the reference couplings squared $\left(g_{H(\mathrm{OP})}^{\mathrm{SM}}\right)^{2}$ are the SM tree-level equivalents:

$$
\begin{aligned}
\left(g_{H Z Z}^{\mathrm{SM}}\right)^{2} & =\left(\frac{e}{s_{\mathrm{w}}} \frac{m_{W}}{c_{\mathrm{w}}^{2}}\right)^{2}, \\
\left(g_{H W W}^{\mathrm{SM}}\right)^{2} & =\left(\frac{e}{s_{\mathrm{w}}} m_{W}\right)^{2}, \\
\left(g_{H f \bar{f}}^{\mathrm{SM}}\right)^{2} & =\left(\frac{1}{2} \frac{e}{s_{\mathrm{w}}} \frac{m_{f}}{m_{W}}\right)^{2},
\end{aligned}
$$

where $m_{W}$ and $m_{f}$ denote the masses of the $W$ boson and fermion $f$, respectively. $\left(g_{H^{\prime} H Z}^{\mathrm{ref}}\right)^{2}$ is defined in Eq. (3) above.

The effective couplings $\left(g_{h_{k}(\mathrm{OP})}^{\text {model }} / g_{H(\mathrm{OP})}^{\mathrm{SM}}\right)^{2}$, with $\mathrm{OP}=Z \gamma, \gamma \gamma$, are loop-induced. They can be defined via

$$
\left(\frac{g_{h_{k}(\mathrm{OP})}^{\text {model }}}{g_{H(\mathrm{OP})}^{\mathrm{SM}}}\right)^{2}=\frac{\Gamma_{h_{k} \rightarrow \mathrm{OP}}^{\text {model }}\left(m_{h_{k}}\right)}{\left.\Gamma_{H \rightarrow \mathrm{OP}}^{\mathrm{SM}}\left(m_{H}\right)\right|_{m_{H}=m_{h_{k}}}}
$$

There is a choice of definition of the Higgs-gluon-gluon effective coupling squared $\left(g_{h_{k}(\mathrm{OP})}^{\text {model }} / g_{H g g}^{\mathrm{SM}}\right)^{2}$. It can either be defined via decay widths:

$$
\left(\frac{g_{h_{k}(\mathrm{OP})}^{\text {model }}}{g_{H g g}^{\mathrm{SM}}}\right)^{2}=\frac{\Gamma_{h_{k} g g}^{\text {model }}\left(m_{h_{k}}\right)}{\left.\Gamma_{H \rightarrow \mathrm{OP}}^{\mathrm{SM}}\left(m_{H}\right)\right|_{m_{H}=m_{h_{k}}}}
$$

or via partonic cross sections:

$$
\left(\frac{g_{h_{k}(\mathrm{OP})}^{\text {model }}}{g_{H g g}^{\mathrm{SM}}}\right)^{2}=R_{g g}^{h_{k}} .
$$


In general, the input option whichinput=effC should only be used when both definitions result in very similar values for $\left(g_{h_{k}(\mathrm{OP})}^{\text {model }} / g_{H g g}^{\mathrm{SM}}\right)^{2}$. However, in certain circumstances, this condition can be relaxed. For example, in a model in which the LEP searches for Higgs bosons decaying into hadrons are not relevant, the normalised gluon-gluon-Higgs effective coupling can be defined solely by Eq. (14). Conversely, if the Tevatron gluon fusion Higgs production mechanism is not relevant, the normalised gluon-gluon-Higgs effective coupling can be defined solely by Eq. (13).

The normalised LEP cross sections are calculated from the normalised effective couplings using the relations:

$$
\begin{aligned}
R_{\sigma}\left(e^{+} e^{-} \rightarrow h_{k} Z\right) & =\left(\frac{g_{h_{k} Z Z}^{\text {model }}}{g_{H Z Z}^{\mathrm{SM}}}\right)^{2}, \\
R_{\sigma}\left(e^{+} e^{-} \rightarrow h_{k} h_{i}\right) & =\left(\frac{g_{H^{\prime} H Z}^{\text {model }}}{g_{H^{\prime} H Z}^{\text {ref }}}\right)^{2}, \\
R_{\sigma}\left(e^{+} e^{-} \rightarrow b \bar{b} h_{k}^{\mathrm{CP} \text { even }}\right) & =\left(\frac{g_{s, h_{k} b \bar{b}}^{\text {model }}}{g_{H b \bar{b}}^{\mathrm{SM}}}\right)^{2}, \\
R_{\sigma}\left(e^{+} e^{-} \rightarrow b \bar{b} h_{k}^{\mathrm{CP} \text { odd }}\right) & =\left(\frac{g_{p, h_{k} b \bar{b}}^{\text {model }}}{g_{H b \bar{b}}^{\mathrm{SM}}}\right)^{2}, \\
R_{\sigma}\left(e^{+} e^{-} \rightarrow \tau^{+} \tau^{-} h_{k}^{\mathrm{CP} \text { even }}\right) & =\left(\frac{g_{s, h_{k} \tau^{+} \tau^{-}}^{\text {model }}}{g_{H \tau^{+} \tau^{-}}^{\mathrm{SM}}}\right)^{2}, \\
R_{\sigma}\left(e^{+} e^{-} \rightarrow \tau^{+} \tau^{-} h_{k}^{\mathrm{CP} \text { odd }}\right) & =\left(\frac{g_{p, h_{k} \tau^{+} \tau^{-}}^{\mathrm{model}}}{g_{H \tau^{+} \tau^{-}}^{\mathrm{SM}}}\right)^{2} .
\end{aligned}
$$

The analyses currently in HiggsBounds which use $\sigma_{\text {model }}\left(e^{+} e^{-} \rightarrow b \bar{b} h_{k}\right)$ or $\sigma_{\text {model }}\left(e^{+} e^{-} \rightarrow\right.$ $\tau^{+} \tau^{-} h_{k}$ ) apply only to Higgs bosons which are CP eigenstates.

The partonic Tevatron cross section ratios are calculated by

$$
\begin{aligned}
& R_{g g}^{h_{k}}=\left(\frac{g_{h_{k} g g}^{\text {model }}}{g_{H g g}^{\mathrm{SM}}}\right)^{2}, \\
& R_{b \bar{b}}^{h_{k}}=R_{b g, \bar{b} g}^{h_{k} b, h_{k} \bar{b}}=\left(\frac{g_{s, h_{k} b \bar{b}}^{\text {model }}}{g_{H b \bar{b}}^{\mathrm{SM}}}\right)^{2}+\left(\frac{g_{p, h_{k} b \bar{b}}^{\text {model }}}{g_{H b \bar{b}}^{\mathrm{SM}}}\right)^{2}, \\
& R_{q \bar{q}^{\prime}}^{h_{k} W^{+}}=R_{q^{\prime} \bar{q}}^{h_{k} W^{-}}=\left(\frac{g_{h_{k} W W}^{\text {model }}}{g_{H W W}^{\mathrm{SM}}}\right)^{2}, \\
& R_{q^{\prime \prime} \bar{q}^{\prime \prime}}^{h_{k} Z}=\left(\frac{g_{h_{k} Z Z}^{\text {model }}}{g_{H Z Z}^{\mathrm{SM}}}\right)^{2},
\end{aligned}
$$

where $\left(q, q^{\prime}\right) \in\{(u, d),(c, s)\}$ and $q^{\prime \prime} \in\{u, d, c, s, b\}$. 
The $t \bar{t}$ CP-even Higgs hadronic Tevatron cross section ratio is obtained using

$$
R_{\sigma}\left(p \bar{p} \rightarrow t \bar{t} h_{k}^{\mathrm{CP} \text { even }}\right)=\left(\frac{g_{s, h_{k} t \bar{t}}^{\text {model }}}{g_{H t \bar{t}}^{\mathrm{SM}}}\right)^{2}
$$

The normalised hadronic cross sections for $h_{k}$ production via VBF is calculated using the approximate relation:

$$
R_{\sigma}\left(p \bar{p} \rightarrow h_{k} \text { via } \mathrm{VBF}\right)=R_{\mathrm{VBF}}^{W W}\left(\frac{g_{h_{k} W W}^{\text {model }}}{g_{H W W}^{\mathrm{SM}}}\right)^{2}+R_{\mathrm{VBF}}^{Z Z}\left(\frac{g_{h_{k} Z Z}^{\text {model }}}{g_{H Z Z}^{\mathrm{SM}}}\right)^{2}
$$

where the numbers

$$
\begin{aligned}
R_{\mathrm{VBF}}^{W W} & :=\frac{\sigma_{\mathrm{SM}}(p \bar{p} \rightarrow H \text { via } W W \text { fusion })}{\sigma_{\mathrm{SM}}(p \bar{p} \rightarrow H \text { via } \mathrm{VBF})}=77 \% \\
R_{\mathrm{VBF}}^{Z Z} & :=\frac{\sigma_{\mathrm{SM}}(p \bar{p} \rightarrow H \text { via } Z Z \text { fusion })}{\sigma_{\mathrm{SM}}(p \bar{p} \rightarrow H \text { via } \mathrm{VBF})}=23 \%
\end{aligned}
$$

have been calculated for $p \bar{p}$ collisions with $1.96 \mathrm{TeV}$ centre-of-mass energy using VBFNLO [21]. Including the Higgs mass dependence in the range $70 \mathrm{GeV}<m_{H}<$ $300 \mathrm{GeV}$ would change these proportions by less than $1 \%$. Interference effects also affect the result by less than 1\% (cf. [27]). (See Ref. [3] for a more detailed discussion.).

Within HiggsBounds, the neutral Higgs decay widths to ordinary particles in the effective coupling input option are then calculated by

$$
\begin{aligned}
\Gamma_{h_{k} \rightarrow \mathrm{OP}}^{\text {model }}\left(m_{h_{k}}\right)= & \left.\left(\frac{g_{h_{k}(\mathrm{OP})}^{\text {model }}}{g_{H(\mathrm{OP})}^{\mathrm{SM}}}\right)^{2} \Gamma_{H \rightarrow \mathrm{OP}}^{\mathrm{SM}}\left(m_{H}\right)\right|_{m_{H}=m_{h_{k}}} \\
& \text { for } \mathrm{OP}=W^{+} W^{-}, Z Z, Z \gamma, \gamma \gamma, g g \\
\Gamma_{h_{k} \rightarrow \mathrm{OP}}^{\text {model }}\left(m_{h_{k}}\right)= & \left.\left(\left(\frac{g_{s, h_{k}(\mathrm{OP})}^{\text {model }}}{g_{H(\mathrm{OP})}^{\mathrm{SM}}}\right)^{2}+\left(\frac{g_{p, h_{k}(\mathrm{OP})}^{\text {model }}}{g_{H(\mathrm{OP})}^{\mathrm{SM}}}\right)^{2} \beta_{f}^{-2}\left(m_{h_{k}}\right)\right) \Gamma_{H \rightarrow \mathrm{OP}}^{\mathrm{SM}}\left(m_{H}\right)\right|_{m_{H}=m_{h_{k}}} \\
& \text { for OP }=s \bar{s}, c \bar{c}, b \bar{b}, \tau^{+} \tau^{-},
\end{aligned}
$$

with $\beta_{f}^{2}\left(m_{h_{k}}\right)=1-4 m_{f}^{2} / m_{h_{k}}^{2}$. These are converted to branching ratios via

$$
\mathrm{BR}_{h_{k} \rightarrow \mathrm{OP}}^{\text {model }}\left(m_{h_{k}}\right)=\frac{\Gamma_{h_{k} \rightarrow \mathrm{OP}}^{\text {model }}\left(m_{h_{k}}\right)}{\Gamma_{\text {tot }}\left(h_{k}\right)}
$$

Note that this means that it is especially important for the user to give an accurate $\Gamma_{\text {tot }}\left(h_{k}\right)$ when using this input option.

Using effective couplings as input, the CP properties of the neutral Higgs bosons can be inferred, at least as far as the signal properties considered here are concerned. In 
the present implementation, we set the $\mathrm{CP}$ value of a Higgs $h_{k}$ in the following way:

$$
\begin{array}{ll}
\mathrm{CP}\left(h_{k}\right) \rightarrow+1 & \text { if } \max _{f}\left(\frac{g_{p, h_{k} f \bar{f}}^{\text {model }}}{g_{H f \bar{f}}^{\mathrm{SM}}}\right)^{2}<10^{-16}, \\
\mathrm{CP}\left(h_{k}\right) \rightarrow-1 & \text { if } \max _{f}\left(\frac{g_{s, h_{k} f \bar{f}}^{\text {model }}}{g_{H f \bar{f}}^{\mathrm{SM}}}\right)^{2}<10^{-16}, \\
\mathrm{CP}\left(h_{k}\right) \rightarrow 0 & \text { if neither of the above conditions is met. }
\end{array}
$$

For the implemented set of analyses, the limits on topological cross sections are applicable with high accuracy to models where the Higgs sector can be faithfully parametrised with effective couplings (See Ref. [3] for details).

In summary, users may find it convenient to use the input option whichinput=effC for cases in which Eq. (6), Eqs. (15) - (26), and Eqs. (29) and (30) are valid to a satisfactory level of accuracy. Of course, if not all of the analyses are relevant to the model, some of these relations do not need to hold. We have already discussed that, in the case of a model in which the LEP searches for Higgs bosons decaying into hadrons are not relevant, Eq. (13) does not need to be valid.

\section{Analyses in HiggsBounds 2.0.0}

HiggsBounds contains many of the available observed and expected exclusion limits at 95\% C.L. (preliminary and final results) from LEP and the Tevatron as data tables which are read in by the program during start-up. In order to provide values for $Q_{\text {obs }}(X)$ and $Q_{\text {expec }}(X)$ for continuous Higgs mass values, the program interpolates $Q$-values linearly between neighbouring Higgs mass points. We aim to include not only the most recent analysis in each channel by each collaboration (or analysis combining results from more than one collaboration) but also the most recent result appearing in a paper submitted to the arXiv. For simplicity we use the label 'published' for those results (since results submitted to the arXiv are usually being prepared for publication in a journal or conference proceedings).

In HiggsBounds 2.0.0, in total 81 Higgs search analyses have been implemented consisting of 28 from LEP and 53 from the Tevatron. While many types of analyses have been added for the first time, several Tevatron analyses included in HiggsBounds 1.2.0 have been replaced by updated ones based on more data. For completeness and as a reference for users, a full list of the implemented analyses follows. 
- LEP neutral Higgs analyses (considering the final states):

$$
\begin{array}{ll}
h_{k} Z, h_{k} \rightarrow b b \text { or } h_{k} \rightarrow \tau \tau[2], & h_{k} Z, h_{k} \rightarrow h_{i} h_{i}, h_{i} \rightarrow b b[2], \\
h_{k} Z, h_{k} \rightarrow \text { anything [4], } & h_{k} Z, h_{k} \rightarrow h_{i} h_{i}, h_{i} \rightarrow \tau \tau[2], \\
h_{k} Z, h_{k} \rightarrow \text { invisible [28-31], } & h_{k} h_{i}, h_{k, i} \rightarrow b b[2], \\
h_{k} Z, h_{k} \rightarrow \gamma \gamma[32], & h_{k} h_{i}, h_{k, i} \rightarrow \tau \tau[2], \\
h_{k} Z, h_{k} \rightarrow \text { hadrons }{ }^{4}, & h_{k} h_{i}, h_{k} \rightarrow h_{i} h_{i}, h_{i} \rightarrow b b[2], \\
b \bar{b} h_{k} \rightarrow b \bar{b} b \bar{b}, h_{k} \text { CP even or odd, [33], } & h_{k} h_{i}, h_{k} \rightarrow h_{i} h_{i}, h_{i} \rightarrow \tau \tau[2], \\
b \bar{b} h_{k} \rightarrow b \bar{b} \tau \tau, h_{k} \text { CP even or odd, [33,34], } & h_{k} h_{i}, h_{k} \rightarrow b b, h_{i} \rightarrow \tau \tau[2], \\
\tau \tau h_{k} \rightarrow \tau \tau \tau \tau, h_{k} \text { CP even or odd, [33]. } &
\end{array}
$$

- Tevatron single topology neutral Higgs analyses (considering the final states):

$$
\begin{array}{ll}
Z h_{k} \rightarrow l l b \bar{b}[39-41], & \text { single } h_{k} \rightarrow W W[42,5], \\
W h_{k} \rightarrow l \nu b \bar{b}[43-46], & \text { single } h_{k} \rightarrow \tau \tau[47-50], \\
W h_{k} \rightarrow 3 W,[51,52], & \text { single } h_{k} \rightarrow Z \gamma,[7], \\
b h_{k} \rightarrow 3 b \text { jets }[53-55], & t \bar{t} h_{k} \rightarrow t \bar{t} b \bar{b}[6], \\
b h_{k} \rightarrow b \tau \tau[56-58] . &
\end{array}
$$

- Tevatron SM Higgs combined analyses considering the final states (schematic):

$$
\begin{aligned}
& V h_{k} \rightarrow b \bar{b}+E_{T}^{\text {miss }} \text { with } V \in\{W, Z\}[59-62], \\
& V h_{k} \rightarrow V V V \rightarrow l^{ \pm} l^{ \pm}+X \text { with } l \in\{e, \mu\} \text { and } V \in\{W, Z\}[63], \\
& h_{k}+X \rightarrow W W+X[64-70], \\
& h_{k}+X \rightarrow \tau \tau+X[71-73], \\
& h_{k}+X \rightarrow b b+X[74], \\
& h_{k}+X \rightarrow \gamma \gamma+X[75-77], \\
& h_{k}+X \text { and various Higgs decays [78-85]. }
\end{aligned}
$$

- LEP/Tevatron charged Higgs analyses:

$$
\begin{aligned}
& e^{+} e^{-} \rightarrow H^{+} H^{-} \rightarrow 4 \text { jets }[86,87], \\
& e^{+} e^{-} \rightarrow H^{+} H^{-} \rightarrow \tau \nu \tau \nu[87], \\
& p \bar{p} \rightarrow t \bar{t}, t \rightarrow H^{+} b, H^{+} \rightarrow c s \text { (\& c.c.) }[88,89], \\
& p \bar{p} \rightarrow t \bar{t}, t \rightarrow H^{+} b, H^{+} \rightarrow \tau \nu \text { (\& c.c.) }[88] .
\end{aligned}
$$

$\overline{4}$ Combination based on $[35-38]$ 
Internally, HiggsBounds uses a number of predictions for SM quantities for the Higgs sector[19-24,90-107] to convert between experimental limits with different normalisations.

Some of the analyses have been performed under certain model assumptions. Typical assumptions are, likeness to the SM, or models which fulfil $\mathrm{BR}\left(t \rightarrow W^{+} b\right)+\mathrm{BR}(t \rightarrow$ $\left.H_{i}^{+} b\right)=1$, or Higgs bosons with certain CP properties. In these cases, HiggsBounds determines for a given model scenario which analyses can be applied to which Higgs boson.

In order to decide whether a model scenario is sufficiently 'SM-like' to be compared with an analysis carried out under SM assumptions, we use the following test. Firstly, for each of the $N_{\mathrm{CS}}$ distinct production cross sections $\sigma_{\text {model }}\left(P_{i}(h)\right)$ and the $N_{\mathrm{BR}}$ distinct decay branching ratios $\mathrm{BR}\left(h \rightarrow F_{k}\right)$ which appear in the list of $M$ signal topologies in that particular analysis, we determine the normalised mean value and the deviation from the mean:

$$
\begin{aligned}
\bar{s} & =\frac{1}{N_{\mathrm{CS}}} \sum_{i=1}^{N_{\mathrm{CS}}} s_{i}, & \bar{b} & =\frac{1}{N_{\mathrm{BR}}} \sum_{k=1}^{N_{\mathrm{BR}}} b_{k}, \\
\delta s_{i} & =s_{i}-\bar{s}, & \delta b_{k} & =b_{k}-\bar{b}, \\
\text { with } s_{i} & =\frac{\sigma_{\text {model }}\left(P_{i}(h)\right)}{\sigma_{\mathrm{SM}}\left(P_{i}(H)\right)}, & b_{k} & =\frac{\mathrm{BR}_{\text {model }}\left(h \rightarrow F_{k}\right)}{\mathrm{BR}_{\mathrm{SM}}\left(H \rightarrow F_{k}\right)} .
\end{aligned}
$$

The parameter point is considered 'SM-like' for this particular analysis if the relative deviations of all individual production and decay combinations $(i, k)$ from the mean $\bar{s} \bar{b}$ stay below a preset bound $\epsilon$ :

$$
\max _{i, k}\left|\frac{\delta s_{i}}{\bar{s}}+\frac{\delta b_{k}}{\bar{b}}+\frac{\delta s_{i} \delta b_{k}}{\bar{s} \bar{b}}\right|<\epsilon .
$$

By default, $\epsilon=2 \%$, i.e. the predictions for the different topological cross sections, normalised to the SM values, are required to result in the same common scale factor with at least $2 \%$ accuracy. This method is conservative and errs on the side of caution, but can occasionally be overly restrictive.

After ensuring that a certain parameter point passes the SM-likeness test for this analysis, the normalised theoretical cross section $Q_{\text {model }}$ is calculated simply by

$$
Q_{\text {model }}=\bar{s} \bar{b}
$$

If two or more neutral Higgs bosons have similar masses, then HiggsBounds allows the possibility of adding up their cross sections. If the mass difference between two neutral Higgs bosons is less than delta_Mh_LEP (or delta_Mh_TEV), then the full LEP 
(or Tevatron) cross sections will be added. The normalisation factor is calculated at the average mass of the Higgs bosons involved. Note that:

delta_Mh_LEP and delta_Mh_TEV are set to zero by default.

To switch this feature on, the user should change these variables in the code and recompile (see the readme files about where to find these variables in the code). This should only be done if the user is confident that interference effects are small. We would recommend that delta_Mh_LEP $\leq 2 \mathrm{GeV}$ and delta_Mh_TEV $\leq 10 \mathrm{GeV}$. For analyses which require a SM-likeness test, the full cross sections are not added, regardless of the values of delta_Mh_LEP and delta_Mh_TEV.

This feature can be very useful in, for example the $m_{h}^{\max }$ scenario of the MSSM [108], where a CP even Higgs boson and the CP odd Higgs boson can have very similar masses but, naturally, they do not mix. In this case, the cross sections are commonly added, such as for example in [53] and [54].

\section{New HiggsBounds Operating Instructions}

Features which are new in version 2.0 .0 will be labelled with the symbol: *.

There are three formats in which the program HiggsBounds can be used:

- Library of subroutines

- Command-line version

- Online version

The most widely applicable format of HiggsBounds is the command-line version, since this reads all the model data from text files and thus this model data can be generated using any package the user wishes. The library of HiggsBounds subroutines allows HiggsBounds to be called within other programs. If the user just wishes to check a few parameter points, the online version provides quick access to all the functionality of HiggsBounds, without the need to install the code.

The HiggsBounds code, the online version and documentation can all be found at the URL http://projects.hepforge.org/higgsbounds/ . ${ }^{5}$

The HiggsBounds code is provided in either Fortran 77 or Fortran 90. Both codes provide exactly the same functionality and have exactly the same operating instructions. In fact, the Fortran 77 and Fortran 90 versions of the HiggsBounds subroutines can even be called within codes written in Fortran 90 and Fortran 77, respectively. Both codes have also been tested with a variety of Fortran compilers, including the

$\overline{5}$ The former website www.ippp.dur.ac.uk/HiggsBounds should redirect to the new one. 
free gnu compilers which accompany most Linux distributions. Therefore, the user may install either code and the difference will only be apparent if the user wishes to examine the structure of the code ${ }^{6}$.

The library of subroutines, the command-line version and the online version share a common set of features, which we will describe first. We will then give operating instructions for each of these three HiggsBounds formats individually.

\subsection{Common features: Input}

HiggsBounds requires five types of input:

- the number of neutral Higgs bosons in the model under study (nHzero)

- the number of singly, positively charged Higgs bosons in the model under study (nHplus)

- the set of experimental analyses which should be considered (whichanalyses)

- the theoretical predictions of the model under study (a set of input arrays)

- the format of these theoretical predictions (whichinput)

Tab. 1 contains further information on the variable nHzero and nHplus, and the possible values of whichanalyses are described in Tab. 2. Note that the option whichanalyses='singH' should only be used if neither processes involving Higgs pair production at LEP nor processes involving the $h_{j} \rightarrow h_{i} h_{i}$ decay are relevant. However, if these conditions are met, this option can save significantly on computing time.

HiggsBounds expects the theoretical input to be in one of three formats, labelled by the variable whichinput. These formats were described in detail in Sect. 3 and are briefly summarised in Tab. 3. In Tab. 4, Tab. 5, Tab. 6 and Tab. 7 we assign names to all of the possible input arrays (each array is defined in terms of the notation used in Sect. 3). These names will prove useful when we describe the input requirements of each version of HiggsBounds individually.

\begin{tabular}{lll} 
nHzero & nHplus * & (integer) \\
\hline $0-9$ & $0-9$ & This is the default range. \\
& & It can easily be extended by the user if required.
\end{tabular}

Table 1

The possible values of the variable nHzero, which labels the number of neutral Higgs bosons in the model under study and the variable nHplus, which labels the number of singly, positively charged Higgs.

6 The maintenance of two separate codes is primarily intended to provide an efficient way for the authors to confirm that each update is free from implementation errors. 


\begin{tabular}{ll} 
whichanalyses & $($ character $(\mathrm{LEN}=5))$ \\
\hline LandT & both LEP and Tevatron analyses \\
onlyL & only LEP analyses \\
onlyT & only Tevatron analyses \\
onlyP & only published analyses (defined as analyses with an arXiv number) \\
singH & only analyses for processes involving one Higgs boson
\end{tabular}

Table 2

The possible values of the variable whichanalyses, which indicates which subset of experimental analyses will be considered by HiggsBounds.

\begin{tabular}{ll} 
whichinput & $($ character $(\mathrm{LEN}=4))$ \\
\hline effC & $\begin{array}{l}\text { Masses, total decay widths, } \\
\text { ratios of effective couplings squared, some branching ratios. }\end{array}$ \\
part & Masses, total decay widths, ratios of LEP cross sections, \\
& mainly ratios of partonic Tevatron cross sections, branching ratios. \\
hadr & Masses, total decay widths, ratios of LEP cross sections, \\
& ratios of hadronic Tevatron cross sections, branching ratios.
\end{tabular}

Table 3

The possible values of the variable whichinput, which indicates the format of the theoretical predictions provided by the user for the neutral Higgs sector. (See Sect. 3 for a more detailed description of each of these settings). 


\begin{tabular}{|c|c|c|c|}
\hline input arrays & \multicolumn{3}{|c|}{ (double precision) } \\
\hline Mh (nHzero) & $m_{h_{i}}$ & in $\mathrm{GeV}$ & \\
\hline MhGammaTot (nHzero) & $\Gamma_{\text {tot }}\left(h_{i}\right)$ & in $\mathrm{GeV}$ & \\
\hline Mhplus (nHplus) * & $m_{H_{i}^{ \pm}}$ & in $\mathrm{GeV}$ & \\
\hline MhplusGammaTot (nHplus) * & $\Gamma_{\text {tot }}\left(H_{i}^{ \pm}\right)$ & in $\mathrm{GeV}$ & \\
\hline g2hjss_s (nHzero) * & $\left(\frac{g_{s, h_{j}(\mathrm{OP})}^{\text {model }}}{g_{H(\mathrm{OP})}^{\mathrm{SM}}}\right)^{2}$ & $\mathrm{OP}=$ & $s \bar{s}$ \\
\hline g2hjcc_s (nHzero)* & & & $c \bar{c}$ \\
\hline g2hjbb_s (nHzero) * & & & $b \bar{b}$ \\
\hline g2hjtoptop_s (nHzero) * & & & $t \bar{t}$ \\
\hline g2hjtautau_s (nHzero)* & & & $\tau^{+} \tau^{-}$ \\
\hline g2hjss_p(nHzero) * & $\left(\frac{g_{p, h_{j}(\mathrm{OP})}^{\mathrm{model}}}{g_{H(\mathrm{OP})}^{\mathrm{SM}}}\right)^{2}$ & $\mathrm{OP}=$ & $s \bar{s}$ \\
\hline g2hjcc_p (nHzero) * & & & $c \bar{c}$ \\
\hline g2hjbb_p (nHzero) * & & & $b \bar{b}$ \\
\hline g2hjtoptop_p (nHzero) * & & & $t \bar{t}$ \\
\hline g2hjtautau_p (nHzero) * & & & $\tau^{+} \tau^{-}$ \\
\hline g2hjWW(nHzero) & $\left(\frac{g_{h_{j}(\mathrm{OP})}^{\text {model }}}{g_{H(\mathrm{OP})}^{\mathrm{SM}}}\right)^{2}$ & $\mathrm{OP}=$ & $W^{+} W^{-}$ \\
\hline g2hjZZ (nHzero) & & & $Z Z$ \\
\hline g2hjZga (nHzero) * & & & $Z \gamma$ \\
\hline g2hjgaga (nHzero) & & & $\gamma \gamma$ \\
\hline g2hjgg (nHzero) & & & $g g$ \\
\hline g2hjhiZ(nHzero,nHzero) & $\left(\frac{g_{h_{j} h_{i} Z}^{\text {model }}}{g_{H H^{\prime} Z}^{\text {ref }}}\right)^{2}$ & & \\
\hline
\end{tabular}

Table 4

Input arrays for model predictions for effective normalised squared couplings recognised by HiggsBounds. The size of each array is given in brackets in the first column. See Sect. 3 for the description of the notation used in the second column. 


\begin{tabular}{|c|c|c|c|}
\hline \multirow[t]{3}{*}{ CP_value (nHzero) *, } & where CP_value(i) & $=-1$ & if $i$ th neutral Higgs is CP-odd \\
\hline & & $=0$ & if $i$ th neutral Higgs has mixed CP \\
\hline & & $=1$ & if $i$ th neutral Higgs is CP-even \\
\hline
\end{tabular}

Table 5

Input array to specify the CP properties of each of the neutral Higgs bosons predicted by the model. The size of the array is given in brackets in the first column.

\begin{tabular}{|c|c|c|c|}
\hline BR_hjss (nHzero) * & $\mathrm{BR}\left(h_{j} \rightarrow \mathrm{OP}\right)$ & $\mathrm{OP}=$ & $s \bar{s}$ \\
\hline BR_hjcc (nHzero) * & & & $c \bar{c}$ \\
\hline BR_hjbb (nHzero) & & & $b \bar{b}$ \\
\hline BR_hjtautau (nHzero) & & & $\tau^{+} \tau^{-}$ \\
\hline BR_hjWW (nHzero) & & & $W^{+} W^{-}$ \\
\hline BR_hjZZ (nHzero) & & & $Z Z$ \\
\hline BR_hjZga (nHzero) * & & & $Z \gamma$ \\
\hline BR_hjgaga (nHzero) & & & $\gamma \gamma$ \\
\hline BR_hjgg (nHzero) * & & & $g g$ \\
\hline BR_hjinvisible (nHzero) * & $\mathrm{BR}\left(h_{j} \rightarrow\right.$ invisible & & \\
\hline BR_hjhihi (nHzero, nHzero) & $\mathrm{BR}\left(h_{j} \rightarrow h_{i} h_{i}\right)$ & & \\
\hline BR_tWpb* & $\mathrm{BR}\left(t \rightarrow W^{+} b\right)$ & & \\
\hline BR_tHpjb (nHplus) * & $\mathrm{BR}\left(t \rightarrow H_{j}^{+} b\right)$ & & \\
\hline BR_Hpjcs (nHplus) * & $\mathrm{BR}\left(H_{j}^{+} \rightarrow \mathrm{OP}\right)$ & $\mathrm{OP}=$ & $c \bar{s}$ \\
\hline BR_Hpj cb (nHplus) * & & & $c \bar{b}$ \\
\hline BR_Hpjtaunu (nHplus) * & & & $\tau^{+} \bar{\nu}_{\tau}$ \\
\hline
\end{tabular}

Table 6

Input arrays for model predictions for branching ratios recognised by HiggsBounds. The size of each array is given in brackets in the first column. See Sect. 3 for the description of the notation used in the second column. The elements of BR_hjhihi are ordered such that $\operatorname{BR\_ hjhihi}(j, i)=B R\left(h_{j} \rightarrow h_{i} h_{i}\right)$. 


\begin{tabular}{|c|c|c|c|}
\hline input arrays cont. & (double & cis & \\
\hline CS_lep_hjZ_ratio(nHzero) & $R_{\sigma}(P)$ & $P=$ & $e^{+} e^{-} \rightarrow h_{j} Z$ \\
\hline CS_lep_bbhj_ratio(nHzero,nHzero) * & & & $e^{+} e^{-} \rightarrow b \bar{b} h_{j}$ \\
\hline CS_lep_tautauhj_ratio(nHzero,nHzero) * & & & $e^{+} e^{-} \rightarrow \tau^{+} \tau^{-} h_{j}$ \\
\hline CS_lep_hjhi_ratio(nHzero,nHzero) & & & $e^{+} e^{-} \rightarrow h_{j} h_{i}$ \\
\hline CS_lep_HpjHmj_ratio(nHzero) * & & & $e^{+} e^{-} \rightarrow H_{j}^{+} H_{j}^{-}$ \\
\hline CS_tev_pp_hj_ratio(nHzero) & & & $p \bar{p} \rightarrow h_{j}$ \\
\hline CS_tev_pp_hjb_ratio(nHzero) & & & $p \bar{p} \rightarrow b h_{j}$ \\
\hline CS_tev_pp_hjW_ratio(nHzero) & & & $p \bar{p} \rightarrow h_{j} W$ \\
\hline CS_tev_pp_hjZ_ratio(nHzero) & & & $p \bar{p} \rightarrow h_{j} Z$ \\
\hline CS_tev_pp_vbf_ratio(nHzero) & & & $p \bar{p} \rightarrow h_{j}$ via $\mathrm{VBF}$ \\
\hline CS_tev_pp_tthj_ratio(nHzero) * & & & $p \bar{p} \rightarrow t \bar{t} h_{j}$ \\
\hline CS_tev_gg_hj_ratio(nHzero) & $R_{n m}^{h_{j}}$ & $n m=$ & $g g$ \\
\hline CS_tev_bb_hj_ratio(nHzero) & & & $b \bar{b}$ \\
\hline CS_tev_ud_hjWp_ratio(nHzero) & $R_{n m}^{h_{j}+W^{+}}$ & $n m=$ & $u \bar{d}$ \\
\hline CS_tev_cs_hjWp_ratio(nHzero) & & & $c \bar{s}$ \\
\hline CS_tev_ud_hjWm_ratio(nHzero) & $R_{n m}^{h_{j}+W^{-}}$ & $n m=$ & $d \bar{u}$ \\
\hline CS_tev_cs_hjWm_ratio(nHzero) & & & $s \bar{c}$ \\
\hline CS_tev_dd_hjZ_ratio(nHzero) & $R_{n m}^{h_{j}+Z}$ & $n m=$ & $d \bar{d}$ \\
\hline CS_tev_uu_hjZ_ratio(nHzero) & & & $u \bar{u}$ \\
\hline CS_tev_ss_hjZ_ratio(nHzero) & & & $s \bar{s}$ \\
\hline CS_tev_cc_hjZ_ratio(nHzero) & & & $c \bar{c}$ \\
\hline CS_tev_bb_hjZ_ratio(nHzero) & & & $b \bar{b}$ \\
\hline CS_tev_bg_hjb_ratio(nHzero) & $R_{n m}^{h_{j}+b, h_{j}+\bar{b}}$ & $n m=$ & $b g, \bar{b} g$ \\
\hline
\end{tabular}

Table 7

Input arrays for model predictions for cross section ratios recognised by HiggsBounds. The size of each array is given in brackets in the first column. The LEP or hadronic Tevatron cross section ratios $R_{\sigma}(P)$ are defined Eq. (3), and the partonic Tevatron cross section ratios $R_{n m}^{h_{j}+y}$ are defined in Eq. (5). 


\subsection{Common features: Output}

HiggsBounds provides the user with four types of output:

- whether the parameter point is excluded at the $95 \%$ C.L. or not (HBresult)

- the reference number of the analysis application $\left(X_{0}\right)$ with the highest statistical sensitivity (chan).

- the number of Higgs bosons which have contributed to the theoretical rate for the corresponding process (ncombined)

- the ratio of the theoretical rate $Q_{\text {model }}$ to the observed limit $Q_{\text {obs }}$ for this process (obsratio).

Tab. 8 shows the possible values of HBresult and obsratio, which are complementary. Tab. 9 and Tab. 10 gives information on chan and ncombined respectively. If the library of subroutines or the command-line versions are used, the key associating the reference numbers with the analysis applications is written in the file Key.dat. In the online version, this information appears on the screen.

\begin{tabular}{lll}
\hline $\begin{array}{l}\text { HBresult } \\
\text { (integer) }\end{array}$ & $\begin{array}{l}\text { obsratio } \\
\text { (double precision) }\end{array}$ \\
\hline 0 & $\geq 1.0$ & parameter point is excluded \\
1 & $<1.0$ & parameter point is not excluded \\
-1 & $\leq 0.0$ & invalid parameter set
\end{tabular}

Table 8

The possible values of the output variables HBresult and obsratio, which indicate whether a parameter point has been excluded at the $95 \%$ C.L. by the experimental results under consideration.

\begin{tabular}{ll} 
chan & (integer) \\
\hline 0-[\# of considered analyses] & $\begin{array}{l}\text { See the file Key.dat for the definition of each } \\
\text { reference number. Key } \cdot \text { dat is automatically } \\
\text { generated when either the command line or the } \\
\text { subroutine version of HiggsBounds are used. }\end{array}$
\end{tabular}

Table 9

Further information about the output variable chan, which stores the reference number of the analysis application with the highest statistical sensitivity. $(0=$ no process applies $)$ 


\begin{tabular}{ll} 
ncombined & (integer) \\
\hline 1-nHzero & $\begin{array}{l}\text { Number of neutral (or singly, positively charged) Higgs bosons } \\
\text { which have contributed to the theoretical rate for this process. }\end{array}$
\end{tabular}

Table 10

Further information about the output variable ncombined.

\subsection{Library of subroutines}

\section{Installation}

The HiggsBounds code can be compiled to form a library of subroutines using the following commands:

./configure

make libHB

A program for which the HiggsBounds subroutines should be used can be compiled and linked to the library by adding $-\mathrm{L}<\mathrm{HB}$ path $>-1 \mathrm{HB}$ to the command line, for example,

gfortran myprog.f90 -o myprog -L<HBpath> -lHB

where $<$ HBpath $>$ is the location of the HiggsBounds library.

The HiggsBounds subroutines make use of the file handles 10, 11, 44, 45 and 87 (i.e. users should avoid using these file handles in the program which calls these subroutines.).

Subroutine initialize_HiggsBounds $\left.{ }^{(}\right)$

The subroutine initialize_HiggsBounds must be called before any other HiggsBounds subroutine. It performs some preparatory operations such as reading in the tables of data. It is called as:

call initialize_HiggsBounds(nHzero, nHplus, whichanalyses)

When using the subroutines in another code, the subroutine initialize_HiggsBounds must be called only once, before any other HiggsBounds subroutine is called.

If the user does not wish to test the neutral Higgs sector with HiggsBounds, then he/she should set nHzero=0. Similarly, if the user does not wish to test the charged Higgs sector with HiggsBounds, then he/she should set nHplus=0. 
Subroutines HiggsBounds_neutral_input_effC ${ }^{\star}$, HiggsBounds_neutral_input_part * and HiggsBounds_neutral_input_hadr *

The neutral Higgs sector input can be passed to HiggsBounds using one of the subroutines HiggsBounds_neutral_input_effC, HiggsBounds_neutral_input_part or HiggsBounds_neutral_input_hadr. They set the value of whichinput to be effC, part and hadr respectively and therefore require different arguments. The use of one of these subroutines is only required if nHzero is non-zero (recall that nHzero is set in subroutine initialize_HiggsBounds). These subroutines are called as:

call HiggsBounds_neutral_input_effC(Mh, MhGammaTot,

\& g2hjss_s,g2hjss_p,g2hjcc_s,g2hjcc_p,g2hjbb_s,g2hjbb_p,

\& g2hjtoptop_s,g2hjtoptop_p,g2hjtautau_s,g2hjtautau_p,

\& g2hjWW,g2hjZZ,g2hjZga,g2hjgaga,g2hjgg,

\& g2hjhiZ,BR_hjinvisible,BR_hjhihi

call HiggsBounds_neutral_input_part(Mh, MhGammaTot, CP_value,

\& CS_lep_hjZ_ratio,CS_lep_bbhj_ratio,CS_lep_tautauhj_ratio,

\& CS_lep_hjhi_ratio,

\& CS_tev_gg_hj_ratio, CS_tev_bb_hj_ratio,

\& CS_tev_bg_hjb_ratio,

\& CS_tev_ud_hjWp_ratio,CS_tev_cs_hjWp_ratio,

\& CS_tev_ud_hjWm_ratio,CS_tev_cs_hjWm_ratio,

\& CS_tev_dd_hjZ_ratio, CS_tev_uu_hjZ_ratio,

\& CS_tev_sS_hjZ_ratio, CS_tev_cc_hjZ_ratio,

\& CS_tev_bb_hjZ_ratio,

\& CS_tev_pp_vbf_ratio, CS_tev_pp_tthj_ratio,

\& BR_hjss,BR_hjcc,BR_hjbb,BR_hjtautau,

\& BR_hjWW,BR_hjZZ,BR_hjZga,BR_hjgaga,BR_hjgg,BR_hjinvisible,

\& BR_hjhihi

call HiggsBounds_neutral_input_hadr(Mh, MhGammaTot, CP_value,

\& CS_lep_hjZ_ratio,CS_lep_bbhj_ratio,CS_lep_tautauhj_ratio,

\& CS_lep_hjhi_ratio,

\& CS_tev_pp_hj_ratio, CS_tev_pp_hjb_ratio,

\& CS_tev_pp_hjW_ratio, CS_tev_pp_hjZ_ratio,

\& CS_tev_pp_vbf_ratio, CS_tev_pp_tthj_ratio,

\& BR_hjss, BR_hjcc,BR_hjbb,BR_hjtautau,

\& BR_hjWW,BR_hjZZ,BR_hjZga,BR_hjgaga,BR_hjgg,BR_hjinvisible,

\& BR_hjhihi

Each of these arguments must be supplied. However, if a branching ratio, effective coupling or cross section is believed to be irrelevant, the corresponding array may be filled with zeros. This will ensure that the value of $Q_{\text {model }}$ for processes involving this quantity will also be zero. For example, in the MSSM, scenarios exist where the 
decay $h_{j} \rightarrow \gamma \gamma$ will certainly not be the process with the highest statistical sensitivity of $Q_{\text {model }} / Q_{\text {expec }}$ and, consequently, it may be convenient to set the arrays g2hjgaga and BR_hjgaga to zero for simplicity in such a case. Note that if a quantity used in a SM-likeness test is set to zero, the model point will fail that SM-likeness test, even if the corresponding SM quantity is very small.

Also, depending on the value given for whichanalyses, some of the input arrays will be ignored within HiggsBounds. For example, if whichanalyses='onlyT', the branching ratio for the Higgs cascade decay $h_{j} \rightarrow h_{i} h_{i}$ will not be relevant. Therefore, setting this array to zero will not affect the HiggsBounds results in this case.

\section{Subroutine HiggsBounds_charged_input *}

The subroutine HiggsBounds_charged_input gives the charged Higgs sector input to HiggsBounds. The use of this subroutine is only required if nHplus is non-zero (recall that nHplus is set in subroutine initialize_HiggsBounds). It is called as:

call HiggsBounds_charged_input(MHplus, MHplusGammaTot

\& CS_lep_HpjHmi_ratio,

\& $\quad$ BR_tWpb,BR_tHpjb,BR_Hpjcs,BR_Hpjcb,BR_Hptaunu )

\section{Subroutine run_HiggsBounds *}

This subroutine performs the main part of the HiggsBounds calculations. It is called as:

call run_HiggsBounds( HBresult, chan, \& obsratio, ncombined )

\section{Subroutine finish_HiggsBounds}

The subroutine finish_HiggsBounds should be called once at the end of the program, after all other HiggsBounds subroutines ${ }^{7}$. It is called as:

call finish_HiggsBounds

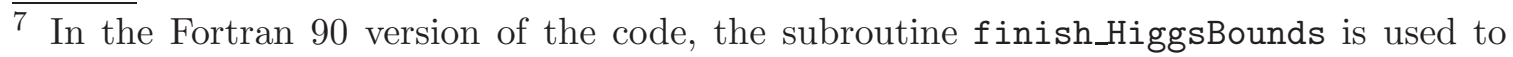
deallocate the allocatable arrays used within HiggsBounds. 
Functions for SM branching ratios, total decay width and cross sections

The HiggsBounds library also allows users access to the SM Higgs branching ratios, total decay width and production cross sections, which are used internally by HiggsBounds. We use SM Higgs branching ratios and total decay width from the program HDECAY 3.4 [19]. The SM hadronic cross sections have been obtained from the TEV4LHC Higgs Working Group [20] (see Tab. 11 for references to the original works) with the exception of the $\sigma^{\mathrm{SM}}(p \bar{p} \rightarrow b g \rightarrow b H)$ and $\sigma^{\mathrm{SM}}(p \bar{p} \rightarrow \bar{b} g \rightarrow \bar{b} H)$ cross sections. The latter cross sections have been calculated with the program HJET 1.3 [22-24] for a set of different cuts on the transverse momentum and pseudo-rapidity of the $b$-quark, which are needed internally in order to apply correctly the results of some Tevatron analyses and which were not available from [20]. From this set, only the cross section without cuts is externally provided.

Also included is a function for the SM top quark decay width into $W^{+} b$ in $\mathrm{GeV}$, which depends on the top quark pole mass in $\mathrm{GeV}$, given at next-to-leading-order, neglecting terms of order $m_{b}^{2} / m_{t}^{2}, \alpha_{s}^{2}$ and $\left(\alpha_{s} / \pi\right) M_{W}^{2} / m_{t}^{2}$, as quoted in [109] (original reference: [110]). This is not used within HiggsBounds, but may prove useful when calculating $\operatorname{BR}\left(t \rightarrow H^{+} b\right)$.

Note that these functions are only valid within certain mass ranges, which are sufficient for the requirements of HiggsBounds. If a function is called with an argument outside this range, it returns a value of -1 .

\section{Examples}

We have provided three example programs which demonstrate the use of the HiggsBounds subroutines. The first example relates to the Fourth Generation Model and is contained in the file example-SM_vs_4thGen.F. This program uses the HiggsBounds functions for the SM branching ratios and SM total decay width to calculate the Higgs decay width and the effective normalised squared couplings in the SM and a simple Fourth Generation Model. This information is then used as input for the subroutine HiggsBounds_neutral_input_effC, which is called once with SM input and once with Fourth Generation Model input. Once the HiggsBounds library has been compiled (using ./configure ; make libHB as described previously), the code example-SM_vs_4thGen.F can be compiled and run with the commands:

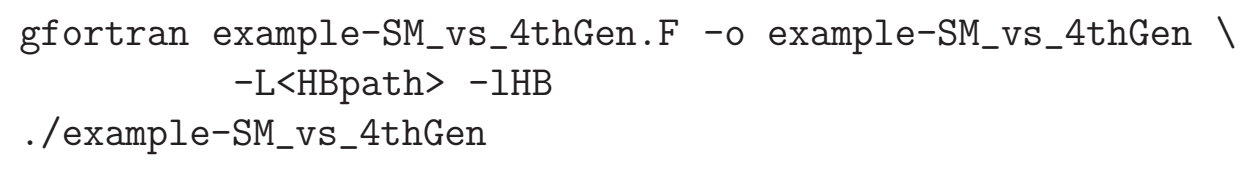

where $<$ HBpath $>$ is the location of the HiggsBounds library. 


\begin{tabular}{|c|c|c|c|}
\hline function & (double precision) & & \\
\hline SMGamma_h (Mh) & $\Gamma_{\text {tot }}^{\mathrm{SM}}\left(h_{i}\right)$ & & {$[19]$} \\
\hline SMBR_Hss (Mh) & $\mathrm{BR}^{\mathrm{SM}}(H \rightarrow \mathrm{OP}), \quad \mathrm{OP}=$ & $s \bar{s}$ & {$[19]$} \\
\hline SMBR_Hcc $($ Mh $)$ & & $c \bar{c}$ & {$[19]$} \\
\hline SMBR_Hbb (Mh) & & $b \bar{b}$ & {$[19]$} \\
\hline SMBR_Htoptop (Mh) & & $t \bar{t}$ & {$[19]$} \\
\hline SMBR_Htautau (Mh) & & $\tau^{-} \tau^{+}$ & {$[19]$} \\
\hline SMBR_Hmumu (Mh) & & $\mu^{-} \mu^{+}$ & {$[19]$} \\
\hline SMBR_HWW (Mh) & & $W^{+} W^{-}$ & {$[19]$} \\
\hline SMBR_HZZ (Mh) & & $Z Z$ & {$[19]$} \\
\hline SMBR_HZgam (Mh) & & $Z_{\gamma}$ & {$[19]$} \\
\hline SMBR_Hgamgam (Mh) & & $\gamma \gamma$ & {$[19]$} \\
\hline SMBR_Hgg (Mh) & & $g g$ & {$[19]$} \\
\hline SMGamma_tWpb (Mtop) * & $\Gamma^{\mathrm{SM}}\left(t \rightarrow W^{+} b\right)$ & & {$[110]$} \\
\hline SMCS_tev_pp_qq_HW (Mh) & $\sigma^{\mathrm{SM}}(P)$ & $p \bar{p} \rightarrow q \bar{q} \rightarrow H W$ & {$[98-100]$} \\
\hline SMCS_tev_pp_qq_HZ (Mh) & & $p \bar{p} \rightarrow q \bar{q} \rightarrow H Z$ & {$[98-100]$} \\
\hline SMCS_tev_pp_gg_H(Mh) & & $p \bar{p} \rightarrow g g \rightarrow H$ & {$[90-97]$} \\
\hline SMCS_tev_pp_bb_H(Mh) & & $p \bar{p} \rightarrow b \bar{b} \rightarrow H$ & {$[101]$} \\
\hline SMCS_tev_pp_vbf_H(Mh) & & $p \bar{p} \rightarrow H$ via $\mathrm{VBF}$ & {$[21,102-104]$} \\
\hline SMCS_tev_pp_ttH $(M h) *$ & & $p \bar{p} \rightarrow t \bar{t} H$ & {$[105-107]$} \\
\hline SMCS_tev_pp_bg_Hb (Mh) & \multicolumn{2}{|c|}{$\sigma^{\mathrm{SM}}(p \bar{p} \rightarrow b g \rightarrow H b)+\sigma^{\mathrm{SM}}(p \bar{p} \rightarrow \bar{b} g \rightarrow H \bar{b})$} & {$[22-24]$} \\
\hline
\end{tabular}

Table 11

SM Higgs branching ratios, total decay widths in units of $\mathrm{GeV}$ and hadronic Tevatron cross sections in units of pb provided as functions by HiggsBounds, together with references. Each function takes a Higgs mass Mh (double precision) as its argument. Also included is the $S M$ top quark decay width into $W^{+} b$ in $\mathrm{GeV}$, which depends on the top quark pole mass in $\mathrm{GeV}$. If a function is called with an argument outside its mass range, it returns a value of -1 .

The files HBwithFH.F and HBwithCPsuperH.f demonstrate the use of the subroutine version of HiggsBounds with the publicly available programs FeynHiggs [8-11] and CPsuperH $[12,13]$, respectively. We refer the reader to the extensive comments contained within these example files for further details. 


\subsection{Command line version}

\section{Installation}

In order to be able to call HiggsBounds from the command line, it should be compiled using the commands

./configure

make

Command line and input file format $\left.{ }^{(}\right)$

In the command-line usage of HiggsBounds, the arrays containing the theoretical model predictions are read from text files. The other options are specified in the command line, which is of the form:

./HiggsBounds <whichanalyses> <whichinput> <nHzero> <nHplus> <prefix>

The variable <prefix $>$ is a string which is added to the front of input and output file names and may include directory names or other identifying information.

Tab. 12 and Tab. 13 describe the contents of each input file. Note that each input file should start with a line number. The input files should not contain any comments or blank lines. The line number identifies the predictions which belong to the same model parameter point in different files. The file BR_H_OP . dat * contains neutral Higgs branching ratios with a SM equivalent (i.e. 'OP'='ordinary particles'), whereas the file BR_H_NP.dat * contains neutral Higgs branching ratios without a SM equivalent (i.e. ' $\mathrm{NP}$ ' $=$ 'new particles') ${ }^{8}$.

Care should be taken with the order of the array elements in the files. This is best illustrated by an example, where we will use $n_{H}=3$. The one dimensional arrays, e.g. Mh, should be given in the order

$$
\operatorname{Mh}(1), \operatorname{Mh}(2), \operatorname{Mh}(3)
$$

8 In previous versions of HiggsBounds, this was equivalent to dividing the neutral Higgs branching ratios between a file containing one type of neutral Higgs and a file containing two types of neutral Higgs bosons. However, the input to HiggsBounds 2.0.0 requires the branching ratio of the Higgs to invisible particles, hence the new filenames. 


\begin{tabular}{|c|c|}
\hline file name & data format \\
\hline MH_GammaTot.dat & $\mathrm{k}$, Mh, MhGammaTot \\
\hline MHplus_GammaTot.dat * & k, Mhplus, MhplusGammaTot \\
\hline \multirow[t]{5}{*}{ effC.dat $(\star)$} & $k, g 2 h j s s_{-} s, g 2 h j s s_{-} p, g 2 h j c c_{-} s, g 2 h j c c \_p$ \\
\hline & g2hjbb_s,g2hjbb_p,g2hjtoptop_s, g2hjtoptop_p \\
\hline & g2hjtautau_s,g2hjtautau_p, \\
\hline & g2hjWW, g2hjZZ, g2hjZga, \\
\hline & $\begin{array}{l}\text { g2hjgaga, } 22 \mathrm{hjgg} \text {, } \\
\text { some elements of g2hjhiZ } \\
\text { (lower left triangle - see example) }\end{array}$ \\
\hline LEP_HZ_CS_ratios.dat & k, CS_lep_hjZ_ratio \\
\hline LEP_H_ff_CS_ratios.dat * & k, CS_lep_bbhj_ratio, CS_lep_tautauhj_ratio \\
\hline LEP_2H_CS_ratios.dat & $\begin{array}{l}\text { k, some elements of CS_lep_hjhi_ratio } \\
\text { (lower left triangle - see example) }\end{array}$ \\
\hline LEP_HpHm_CS_ratios.dat * & k, CS_lep_HpjHmj_ratio \\
\hline TEV_H_Ojet_partCS_ratios.dat & k, CS_tev_gg_hj_ratio, CS_tev_bb_hj_ratio \\
\hline TEV_H_1jet_partCS_ratios.dat & k CS_tev_bg_hjb_ratio \\
\hline TEV_HW_partCS_ratios.dat & $\begin{array}{l}\text { k, CS_tev_ud_hjWp_ratio,CS_tev_cs_hjWp_ratio, } \\
\text { CS_tev_ud_hjWm_ratio,CS_tev_cs_hjWm_ratio }\end{array}$ \\
\hline TEV_HZ_partCS_ratios.dat & $\begin{array}{l}\text { k, CS_tev_dd_hjZ_ratio, CS_tev_uu_hjZ_ratio, } \\
\text { CS_tev_ss_hjZ_ratio, CS_tev_cc_hjZ_ratio, } \\
\text { CS_tev_bb_hjZ_ratio }\end{array}$ \\
\hline TEV_H_vbf_hadCS_ratios.dat & k, CS_tev_pp_vbf_ratio \\
\hline TEV_H_tt_hadCS_ratios.dat * & k, CS_tev_pp_tthj_ratio \\
\hline \multirow[t]{2}{*}{ TEV_1H_hadCS_ratios.dat ${ }^{(\star)}$} & $\begin{array}{l}\text { k, CS_tev_pp_hj_ratio, CS_tev_pp_hjb_ratio, } \\
\text { CS_tev_pp_hjW_ratio,CS_tev_pp_hjZ_ratio, } \\
\text { CS_tev_pp_vbf_ratio }\end{array}$ \\
\hline & CS_tev_pp_tthj_ratio \\
\hline
\end{tabular}

Table 12

Names and data format of all HiggsBounds input files (part I). The right column shows the order of the input data arrays within one line of the input file. For the order within the arrays, see example. $\mathrm{k}$ is the line number. Note that the arrays CS_tev_pp_vbf_ratio, CS_tev_pp_tthj_ratio each appear in two different input files. However, these files will never be required by HiggsBounds simultaneously. 


\begin{tabular}{|c|c|}
\hline file name & data format \\
\hline BR_H_OP . dat * & 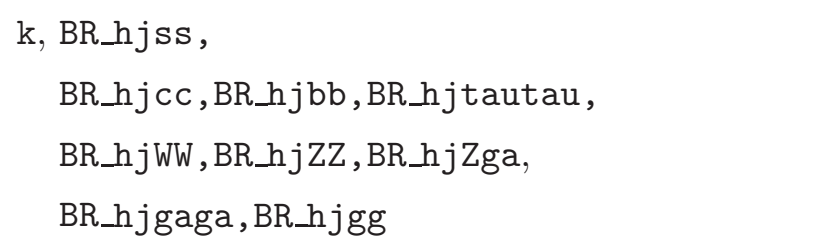 \\
\hline BR_H_NP.dat * & $\begin{array}{l}\mathrm{k}, \mathrm{BR} \_\mathrm{h} \text { jinvisible, some elements of BR_hjhihi } \\
\text { (row by row, without diagonal } \\
\text { - see example) }\end{array}$ \\
\hline BR_t.dat* & $\mathrm{k}, \mathrm{BR} \_\mathrm{tWpb}, \mathrm{BR} \_\mathrm{tHpb}$ \\
\hline BR_Hplus . dat * & k, BR_Hpcs, BR_Hpcb, BR_Hptaunu \\
\hline CP_values.dat * & $\mathrm{k}$, CP_value \\
\hline additional.dat(optional) & $\mathrm{k}, . .$. \\
\hline
\end{tabular}

Table 13

Names and data format of all HiggsBounds input files (part II). The right column shows the order of the input data arrays within one line of the input file. For the order within the arrays, see example. $\mathrm{k}$ is the line number. Note that the arrays CS_tev_pp_vbf_ratio, CS_tev_pp_tthj_ratio each appear in two different input files. However, these files will never be required by HiggsBounds simultaneously. 
However, not all of the elements of the two dimensional arrays are required. Only the lower left triangle (including the diagonal) is required from the arrays g2hjhiZ and lepCS_hjhi_ratio, since they are symmetric, e.g.

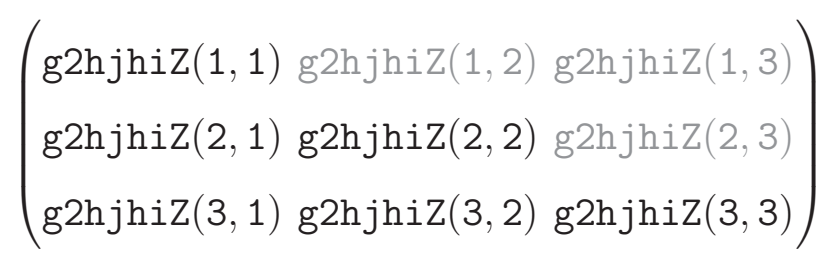

i.e. the elements in the input file should be written in the order

$$
\text { g2hjhiZ(1,1), g2hjhiZ(2,1), g2hjhiZ(2, 2), g2hjhiZ(3,1), }
$$
$\operatorname{ghjhiZ}(3,2), \operatorname{g} 2 h j h i Z(3,3)$.

For the array BR_hjhihi, only the off-diagonal components are required

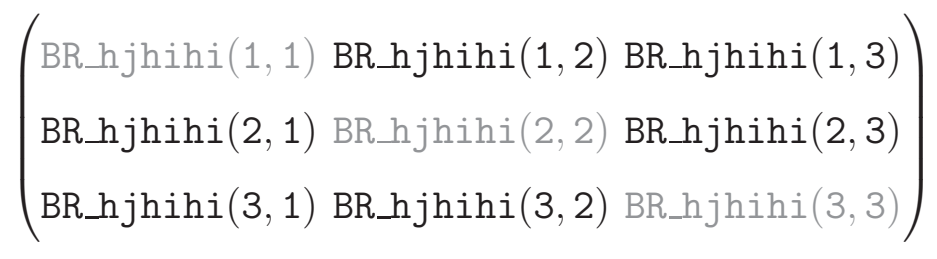

since the diagonal elements are not physical quantities. Therefore, the elements should be written in the order

BR_hjhihi (1,2), BR_hjhihi (1,3), BR_hjhihi $(2,1)$, BR_hjhihi (2,3),

BR_hjhihi (3,1), BR_hjhihi $(3,2)$

in the input file.

The file additional.dat is optional. If it is included, it can have any number of columns greater than 1 (as for the previous files, the first entry on each line should be the line number). It is envisaged that this input file will be particularly useful when parameter scans are performed over a variable which is not required by HiggsBounds but helpful when plotting the results. For example, in the case of the MSSM, additional. dat could be used to store the values of $\tan \beta$.

As in the subroutine version, the command line version of HiggsBounds expects a subset of the total list of input arrays, which depends on the chosen setting of whichinput. The maximal list of files used for each value of whichinput is given in Tab. 2.

As discussed for the subroutine version, some of the arrays will not be relevant for some of the choices for whichanalyses. The command line version of HiggsBounds will consider the list of input files appropriate to the setting whichinput and then only attempt to read any of these input files if the value chosen for whichanalyses means that at least one of the arrays it contains will be directly used. Tab. 15 contains 
a list of which input files are actually relevant to each value of whichanalyses. For example, if whichinput = 'hadr', whichanalyses = 'LandT', nHzero $>0$ and nHplus > 0 then HiggsBounds requires the input files:

MH_GammaTot.dat, MHplus_GammaTot.dat, CP_values.dat, BR_H_NP.dat, BR_H_OP.dat, BR_t.dat, BR_Hplus . dat, LEP_HZ_CS_ratios.dat, LEP_H_ff_CS_ratios.dat, LEP_2H_CS_ratios.dat, LEP_HpHm_CS_ratios.dat, TEV_1H_hadCS_ratios.dat .

However, if whichinput = 'hadr', whichanalyses = 'onlyL', nHzero $>0$ and nHplus > 0 HiggsBounds requires the input files:

MH_GammaTot.dat, MHplus_GammaTot.dat, CP_values.dat, BR_H_NP . dat, BR_H_OP . dat, BR_Hplus . dat, LEP_HZ_CS_ratios.dat, LEP_H_ff_CS_ratios.dat, LEP_2H_CS_ratios.dat, LEP_HpHm_CS_ratios.dat.

As a third example, if whichinput = 'hadr', whichanalyses = 'onlyT', nHzero $>0$ and nHplus > 0 HiggsBounds requires the input files:

MH_GammaTot.dat, MHplus_GammaTot.dat, CP_values.dat, BR_H_NP.dat, BR_H_OP.dat, BR_t.dat, BR_Hplus.dat, TEV_1H_hadCS_ratios.dat .

In each of these three examples, HiggsBounds will also read the file additional.dat

if it exists.

As for the subroutine version, if the user does not require processes involving a particular branching ratio or cross section ratio to be checked by HiggsBounds, that particular array can be filled with zeros.

\section{Output file format}

When the command line version of HiggsBounds is used, the output is written to the file <prefix>HiggsBounds_results . dat. A sample of the output is shown in Fig. 1. The key to the process numbering is written to <prefix>Key.dat.

\section{Examples}

The HiggsBounds package includes a full set of sample input files for the case $n_{H}=$ $3, n_{H^{+}}=1$, contained in the folder example_data. Each filename is prefixed with 


\begin{tabular}{|c|c|c|}
\hline whichinput = 'part', & 'hadr', & 'effC' \\
\hline MH_GammaTot.dat & MH_GammaTot.dat & MH_GammaTot.dat \\
\hline MHplus_GammaTot.dat * & MHplus_GammaTot.dat * & MHplus_GammaTot.dat * \\
\hline BR_H_NP.dat * & BR_H_NP.dat * & $\operatorname{effC} \cdot \operatorname{dat}^{(\star)}$ \\
\hline BR_H_OP . dat * & BR_H_OP . dat * & BR_H_NP.dat * \\
\hline BR_t.dat* & BR_t.dat * & BR_t.dat* \\
\hline BR_Hplus.dat * & BR_Hplus . dat * & BR_Hplus . dat * \\
\hline LEP_HZ_CS_ratios.dat & LEP_HZ_CS_ratios.dat & LEP_HpHm_CS_ratios.dat * \\
\hline LEP_H_ff_CS_ratios.dat * & LEP_H_ff_CS_ratios.dat * & additional.dat \\
\hline LEP_2H_CS_ratios.dat & LEP_2H_CS_ratios.dat & \\
\hline LEP_HpHm_CS_ratios.dat * & LEP_HpHm_CS_ratios.dat * & \\
\hline TEV_H_Ojet_partCS_ratios.dat & TEV_1H_hadCS_ratios.dat ${ }^{(\star)}$ & \\
\hline TEV_H_1jet_partCS_ratios.dat & CP_values.dat * & \\
\hline TEV_HW_partCS_ratios.dat & additional.dat & \\
\hline \multicolumn{3}{|l|}{ TEV_HZ_partCS_ratios.dat } \\
\hline \multicolumn{3}{|l|}{ TEV_H_vbf_hadCS_ratios.dat } \\
\hline \multicolumn{3}{|l|}{ TEV_H_tt_hadCS_ratios.dat * } \\
\hline \multicolumn{3}{|l|}{ CP_values.dat * } \\
\hline additional.dat & & \\
\hline
\end{tabular}

Table 14

The list of possible input files for each value of whichinput. Note that some input files may not be relevant, depending on the values of whichanalyses, nHzero and nHplus. In this case, they are not required. See Tab. 15 and Tab. 16 for more details.

HB_randomtest50points_. To run the command-line version of HiggsBounds with these files as input, use, for example,

./configure

make

./HiggsBounds LandT effC 31 'example_data/HB_randomtest50points_'

where the values of whichanalyses and whichinput can be varied as desired. The setting nHplus $=0$ can be used if the user does not wish to test the charged Higgs sector. E.g.

./HiggsBounds LandT effC 30 'example_data/HB_randomtest50points_' 


\begin{tabular}{|c|c|c|c|c|c|}
\hline \multirow[t]{2}{*}{ name of input file } & \multicolumn{5}{|c|}{$\begin{array}{l}\text { values of whichanalyses } \\
\text { which this file is relevant to }\end{array}$} \\
\hline & LandT & onlyL & onlyT & $\operatorname{singH}$ & onlyP * \\
\hline MH_GammaTot.dat & $\mathrm{y}$ & $\mathrm{y}$ & $\mathrm{y}$ & $\mathrm{y}$ & $\mathrm{y}$ \\
\hline MHplus_GammaTot.dat * & $\mathrm{y}$ & $\mathrm{y}$ & $\mathrm{y}$ & $\mathrm{y}$ & $\mathrm{y}$ \\
\hline effC.dat ${ }^{(\star)}$ & $\mathrm{y}$ & $\mathrm{y}$ & $\mathrm{y}$ & $\mathrm{y}$ & $\mathrm{y}$ \\
\hline LEP_HZ_CS_ratios.dat & $\mathrm{y}$ & $\mathrm{y}$ & & $\mathrm{y}$ & $\mathrm{y}$ \\
\hline LEP_H_ff_CS_ratios . dat * & $\mathrm{y}$ & $\mathrm{y}$ & & $\mathrm{y}$ & $\mathrm{y}$ \\
\hline LEP_2H_CS_ratios.dat & $\mathrm{y}$ & $\mathrm{y}$ & & & $\mathrm{y}$ \\
\hline LEP_HpHm_CS_ratios.dat * & $\mathrm{y}$ & $\mathrm{y}$ & & $\mathrm{y}$ & $\mathrm{y}$ \\
\hline TEV_H_Ojet_partCS_ratios.dat & $\mathrm{y}$ & & $\mathrm{y}$ & $\mathrm{y}$ & $\mathrm{y}$ \\
\hline TEV_H_1jet_partCS_ratios.dat & $\mathrm{y}$ & & $\mathrm{y}$ & $\mathrm{y}$ & $\mathrm{y}$ \\
\hline TEV_HW_partCS_ratios.dat & $\mathrm{y}$ & & $\mathrm{y}$ & $\mathrm{y}$ & $\mathrm{y}$ \\
\hline TEV_HZ_partCS_ratios.dat & $\mathrm{y}$ & & $\mathrm{y}$ & $\mathrm{y}$ & $\mathrm{y}$ \\
\hline TEV_H_vbf_hadCS_ratios.dat & $\mathrm{y}$ & & $\mathrm{y}$ & $\mathrm{y}$ & $\mathrm{y}$ \\
\hline TEV_H_tt_hadCS_ratios.dat * & $\mathrm{y}$ & & $\mathrm{y}$ & $\mathrm{y}$ & $\mathrm{y}$ \\
\hline TEV_1H_hadCS_ratios.dat ${ }^{(\star)}$ & $\mathrm{y}$ & & $\mathrm{y}$ & $\mathrm{y}$ & $\mathrm{y}$ \\
\hline BR_H_OP . dat * & $\mathrm{y}$ & $\mathrm{y}$ & $\mathrm{y}$ & $\mathrm{y}$ & $\mathrm{y}$ \\
\hline BR_H_NP.dat * & $\mathrm{y}$ & $\mathrm{y}$ & $\mathrm{y}$ & $\mathrm{y}$ & $\mathrm{y}$ \\
\hline BR_t.dat * & $\mathrm{y}$ & & $\mathrm{y}$ & $\mathrm{y}$ & $\mathrm{y}$ \\
\hline BR_Hplus.dat * & $\mathrm{y}$ & $\mathrm{y}$ & $\mathrm{y}$ & $\mathrm{y}$ & $\mathrm{y}$ \\
\hline CP_values.dat* & $\mathrm{y}$ & $\mathrm{y}$ & $\mathrm{y}$ & $\mathrm{y}$ & $\mathrm{y}$ \\
\hline additional.dat (optional) & $\mathrm{y}$ & $\mathrm{y}$ & $\mathrm{y}$ & $\mathrm{y}$ & $\mathrm{y}$ \\
\hline
\end{tabular}

Table 15

List of input files, specifying which values of whichanalyses each input file is relevant to (marked by 'y'). Note that some input files may not be relevant, depending on the values of whichinput, nHzero and nHplus. In this case, they are not required. See Tab. 14 and Tab. 16 for more details. 


\begin{tabular}{|c|c|c|}
\hline name of input file & $\begin{array}{l}\text { only relevant if } \\
\text { nHzero > } 0\end{array}$ & $\begin{array}{l}\text { only relevant if } \\
\text { nHplus }{ }^{\star}>0\end{array}$ \\
\hline MH_GammaTot.dat & $\mathrm{y}$ & \\
\hline MHplus_GammaTot.dat * & & $\mathrm{y}$ \\
\hline $\operatorname{effC}$.dat ${ }^{(\star)}$ & $\mathrm{y}$ & \\
\hline LEP_HZ_CS_ratios.dat & $\mathrm{y}$ & \\
\hline LEP_H_ff_CS_ratios . dat * & $\mathrm{y}$ & \\
\hline LEP_2H_CS_ratios.dat & $\mathrm{y}$ & \\
\hline LEP_HpHm_CS_ratios.dat * & & $\mathrm{y}$ \\
\hline TEV_H_Ojet_partCS_ratios . dat & $\mathrm{y}$ & \\
\hline TEV_H_1jet_partCS_ratios.dat & $\mathrm{y}$ & \\
\hline TEV_HW_partCS_ratios.dat & $\mathrm{y}$ & \\
\hline TEV_HZ_partCS_ratios.dat & $\mathrm{y}$ & \\
\hline TEV_H_vbf_hadCS_ratios.dat & $\mathrm{y}$ & \\
\hline TEV_H_tt_hadCS_ratios.dat * & $\mathrm{y}$ & \\
\hline TEV_1H_hadCS_ratios.dat ${ }^{(\star)}$ & $\mathrm{y}$ & \\
\hline BR_H_OP . dat * & $\mathrm{y}$ & \\
\hline BR_H_NP.dat * & $\mathrm{y}$ & \\
\hline BR_t.dat * & & $\mathrm{y}$ \\
\hline BR_Hplus .dat* & & $\mathrm{y}$ \\
\hline CP_values.dat * & $\mathrm{y}$ & \\
\hline additional.dat & (optional) & (optional) \\
\hline
\end{tabular}

Table 16

List of files, showing which relate to the neutral Higgs searches and which relate to the charged Higgs searches. Note that some input files may not be relevant, depending on the values of whichinput and whichanalyses. In this case, they are not required. See Tab. 14 and Tab. 15 for more details. 


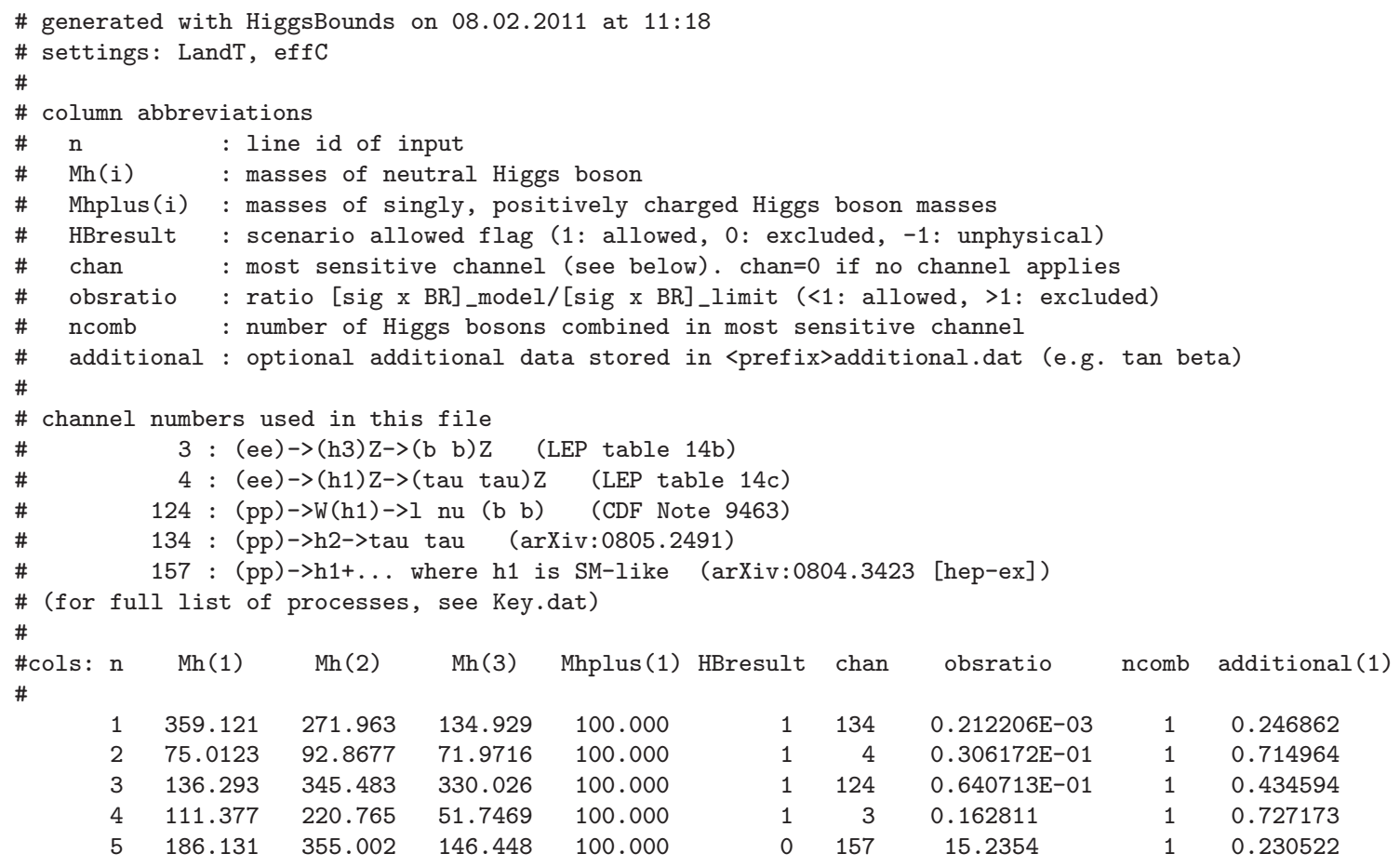

Fig. 1. Sample output file (written to <prefix>HiggsBounds_Results.dat)

\subsection{Online version}

The online version can be reached via the website ${ }^{9}$

http://projects.hepforge.org/higgsbounds/ .

It allows the user to select the required number of neutral Higgs and charged Higgs bosons and then generates a html form accordingly. The values of whichinput and whichanalyses can be chosen and the appropriate theoretical input entered. HiggsBounds will then be called with these settings and the result outputted to screen. The online version contains the additional feature that it notifies the user about the processes with the second and third highest statistical sensitivities and the values of obsratio for these processes. This is designed to give guidance to the user who, for example, wishes to find an excluded region iteratively by adjusting the input quantities.

The website also contains a selection of pre-filled html forms as examples, including entries for the SM, the fermiophobic Higgs model and the MSSM with real and complex parameters.

* There is also the possibility of copying and pasting the results from the online version of FeynHiggs (the 'FeynHiggs User Control Center' [111]) into a box on the

$\overline{9}$ The former website www.ippp.dur.ac.uk/HiggsBounds should redirect to the new one. 
HiggsBounds website for the CP-conserving MSSM. This text is then converted to a filled-in HiggsBounds input html form (which can be further edited by the user if necessary) that can then be immediately submitted to HiggsBounds.

\section{$6 \quad$ Physics Applications}

\subsection{Model scenarios with invisible Higgs decay modes}

The decay of a Higgs boson into stable, weakly interacting neutral particles (invisible Higgs decay) is allowed in a wide variety of models. In the R-parity conserving MSSM with a neutralino $\chi_{1}^{0}$ as the lightest supersymmetric particle (LSP), the decay $h^{0} \rightarrow$ $\chi_{1}^{0} \chi_{1}^{0}$ is dominant in some scenarios $[112,113]$. Invisible Higgs decays can also be important in models with hidden sectors that couple to the Higgs sector [114], models where a Higgs boson can decay into a pair of Majorons [115-117], models with 4th generation neutrinos [118], non-linear supersymmetric models in which the Higgs boson can decay into a Goldstino and a neutralino [119] and some extra dimension models [120-125].

We consider here a simple model in which we vary the branching ratio of the invisible Higgs decay mode, while keeping all other cross sections and decay widths fixed to SM values and assuming that the narrow width approximation holds. This is achieved with the following program:

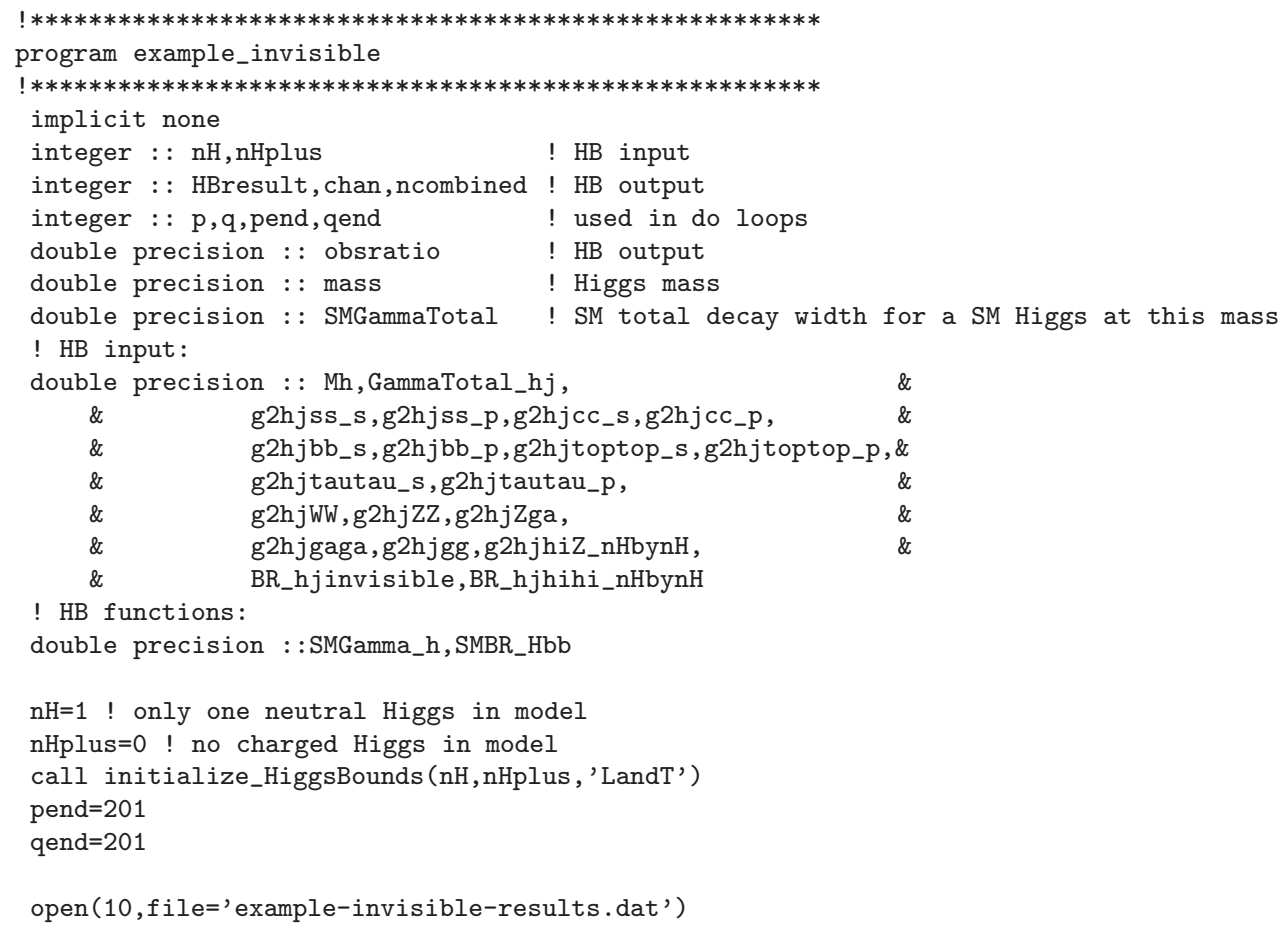




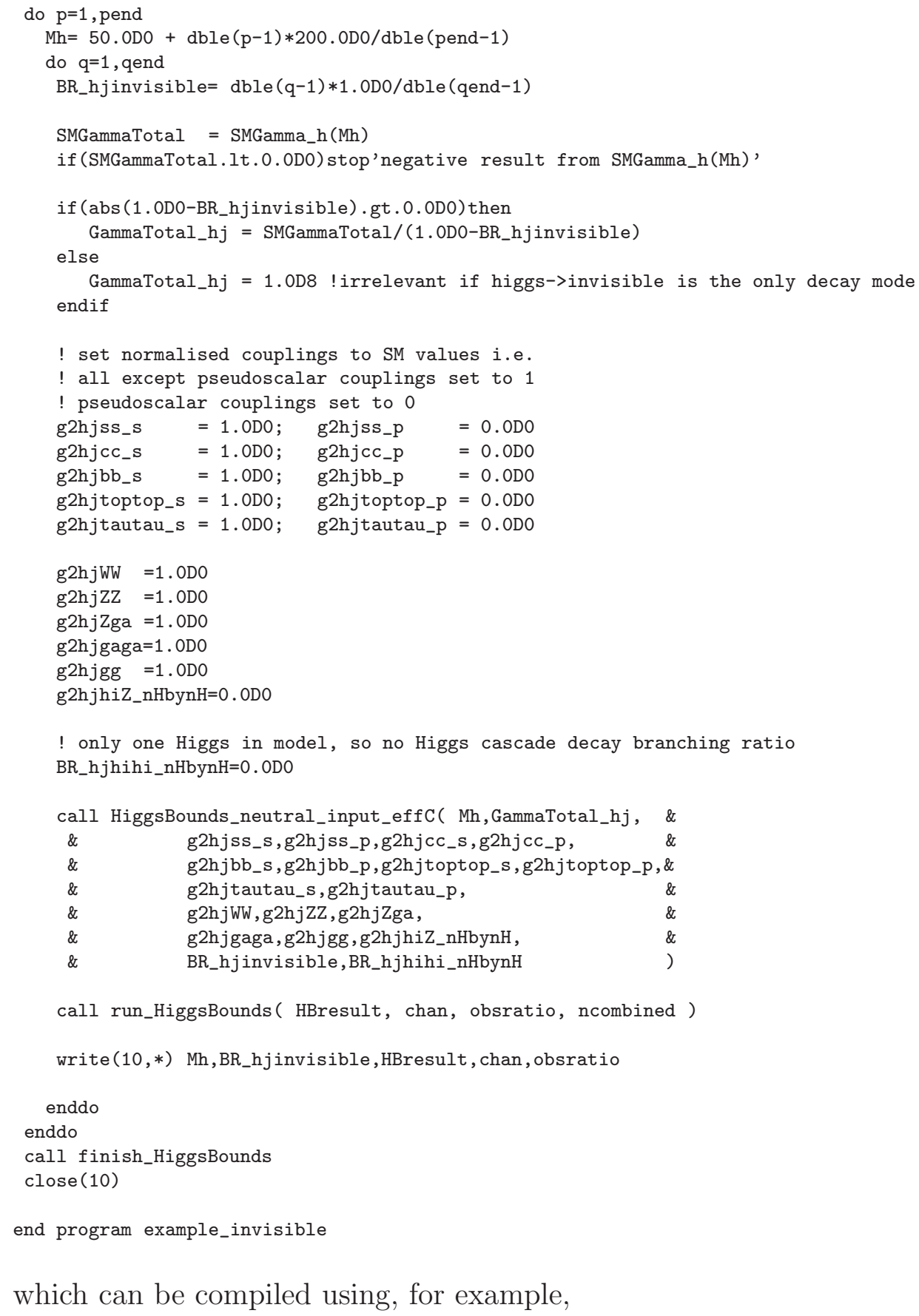

which can be compiled using, for example,

gfortran ./example_invisible.f90 -o ./example_invisible.exe -L<HBdirectorypath> - lHB

\subsubsection{Exclusion}

We show the results from the code displayed above in Fig. 2. In the left panel, we can see that all values of the invisible Higgs branching ratio are excluded almost up to the kinematical limit of LEP. In the right panel, we can see that LEP Higgsstrahlung processes have the highest statistical sensitivity in this region, and therefore were 

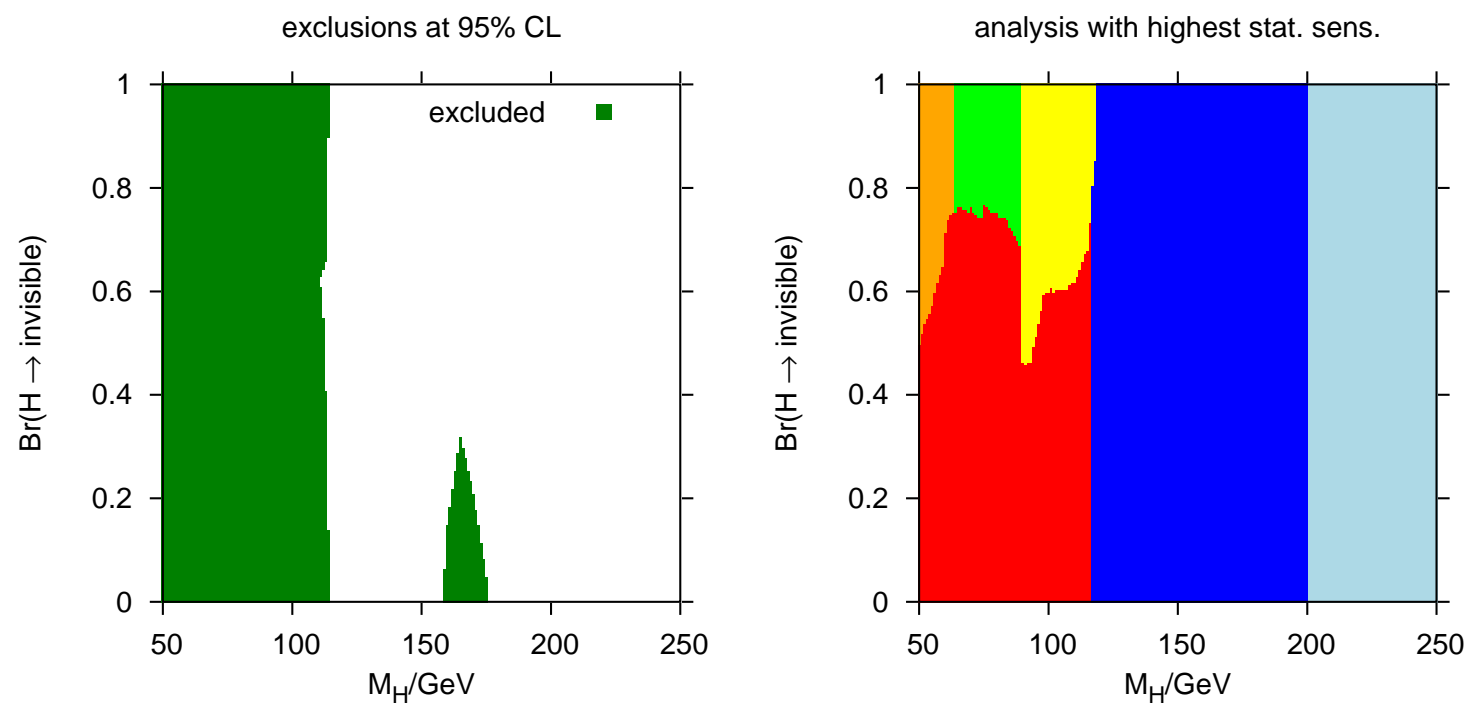

Fig. 2. Parameter space for our toy model, in which we vary the branching ratio of the Higgs decay into invisible particles and the Higgs mass. Higgs production cross sections and all other Higgs decay widths take SM values. Left: parameter points excluded at 95\% C.L. by Higgs searches at LEP and the Tevatron. Green (dark grey) = excluded, White = unexcluded.

Right: Channel with the highest statistical sensitivity at each parameter point.

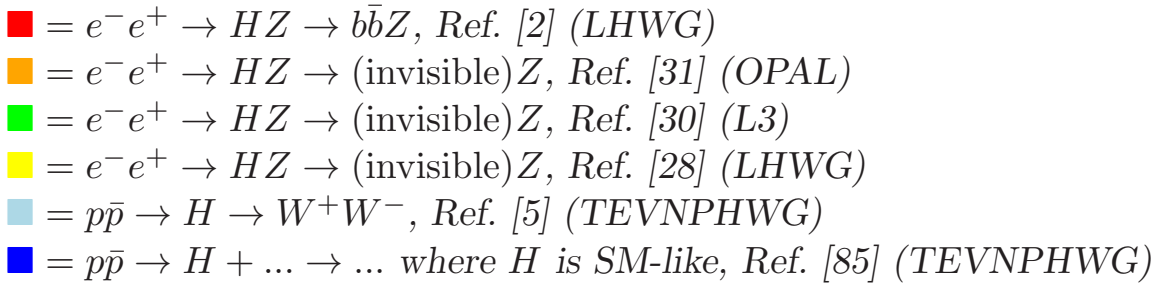

used to obtain this exclusion. For lower values of invisible Higgs branching ratio, the decay mode $H \rightarrow b \bar{b}$ was used, whereas at higher values of $\operatorname{BR}(H \rightarrow$ invisible $)$, Higgsstrahlung topologies which explicitly involve the $H \rightarrow$ invisible decay mode (i.e. searches for a $Z$-boson plus large missing momentum) were used. The LEP Higgs Working Group combined invisible Higgs analysis [28] does not cover the region $M_{H}<$ $90 \mathrm{GeV}$, and therefore the OPAL [31] and L3 [30] analyses are also required.

We can also apply the results from the Tevatron SM Higgs searches directly to this model, since all the relevant cross sections and branching ratios differ from their SM equivalents by a common factor (i.e. the parameter points all pass the SM-likeness test for the Tevatron SM Higgs analyses, as described in Sect. 4). Therefore, the exclusion at 95\% C.L. of a SM Higgs boson between $158 \mathrm{GeV}$ and $175 \mathrm{GeV}$ provided by Ref. [85] translates to a wedge-shaped excluded region in the parameter space of our toy model, extending up to $\mathrm{BR}(H \rightarrow$ invisible $)=0.32$. 

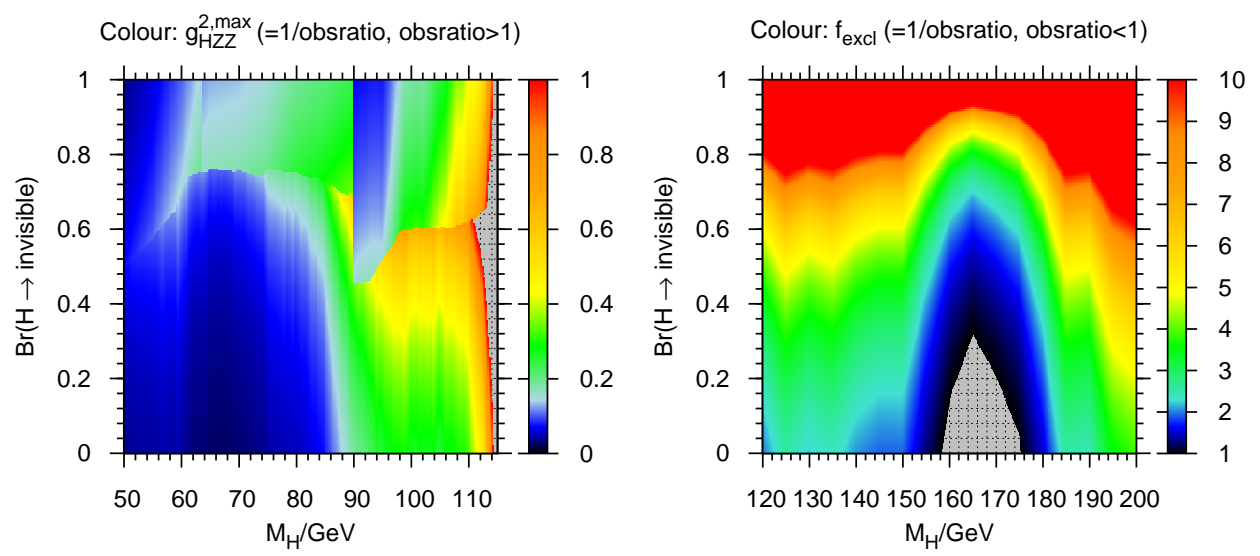

Fig. 3. Subsections of the parameter space for our toy model, in which we vary the branching ratio of Higgs into invisible particles and the Higgs mass. obsratio is the ratio of the theoretical cross section to the observed cross section for the channel with the highest statistical sensitivity, as calculated by the program example_invisible, plotted when obsratio $>1$ (left) or when obsratio $<1$ (right). Left: we interpret 1 /obsratio as the maximum value of $g_{H Z Z}^{2}$ which is consistent with the parameter point being excluded at 95\% C.L. Right: we interpret 1 /obsratio as the factor $f_{\text {excl }}$ by which the measured SM cross section limit must be reduced in order to exclude this parameter point at 95\% C.L. (in this plot, red denotes $f_{\text {excl }} \geq 10$ ).

\subsubsection{Interpreting the variable obsratio}

We can also obtain interesting information about our model from the variable obsratio outputted by the program example_invisible. Recall that obsratio is the ratio of the theoretical cross section to the observed cross section for the channel with the highest statistical sensitivity at each parameter point $Q_{\text {model }}\left(X_{0}\right) / Q_{\text {obs }}\left(X_{0}\right)$, as discussed in Sect. 5. Roughly speaking, this variable gives an idea of 'how strong' the exclusion of a particular parameter point is. We shall now demonstrate two possible ways in which this variable can be used to obtain additional insights into our toy model. We will first discuss the excluded low mass region (with obsratio $>1$ ) and then we will discuss the unexcluded intermediate mass region (with obsratio $<1$ ).

In models with extended Higgs sectors, such as the MSSM, the Higgs couplings to $Z$-bosons frequently obey the sum rule $\sum_{i=1}^{n_{H}^{0}} g_{h_{i} Z Z}^{2}=1$, and therefore the $h_{i}-Z$ - $Z$ couplings in these models are suppressed with respect to the SM coupling. It is therefore interesting to consider the extent to which the parameter space of our toy model can still be excluded if we suppress the $H-Z-Z$ coupling. We can interpret the inverse of the obsratio calculated by the program example_invisible as the maximum $g_{H Z Z}^{2}$ which will still allow the parameter point to be excluded at $95 \%$ by the LEP Higgs search results (considering Higgsstrahlung processes only). 1/obsratio is plotted in Fig. 3(left) in the low Higgs mass region, for obsratio $>1$. In this plot, we can see that, for example, a Higgs boson with $M_{H}=95 \mathrm{GeV}$ and $\mathrm{BR}(H \rightarrow$ invisible $)=0.5$ (and all other decay widths as in SM) can not be excluded by the LEP Higgsstrahlung 
searches if $g_{H Z Z}^{2}<0.5$.

We shall now look at 1 /obsratio in the unexcluded part of the intermediate Higgs mass range, where the Tevatron SM Higgs analysis Ref. [85] has the highest statistical sensitivity. This is shown in Fig. 3(right), for obsratio $<1$. In this mass range, it is interesting to interpret 1 /obsratio as the factor $f_{\text {excl }}$ by which the current SM cross section limit must be lowered in order to obtain a $95 \%$ exclusion in our toy model. Therefore, if, for example, a future Tevatron analysis can lower the current limit by $10 \%$ at $M_{H}=165 \mathrm{GeV}$, this will allow $\mathrm{BR}(H \rightarrow$ invisible $)$ up to 0.38 to be excluded in our toy model at this Higgs mass. In this way, we can get an impression of which areas of parameter space are likely to be testable in the near future and which areas will require much more data.

\subsection{Constraints on the Randall-Sundrum scalar sector}

The Randall-Sundrum (RS) Model, RS1, considers spacetime a slice of 5d anti-deSitter space with two boundaries, the IR brane (our 4d spacetime) and the UV brane [126]. This configuration yields an explanation for the hierarchy problem, i.e. why the mass scale of electroweak physics is so much smaller than the Planck scale $M_{\mathrm{Pl}}$. The spacetime metric can be written as

$$
d s^{2}=e^{-2 k r_{c} y} \eta_{\mu \nu} d x^{\mu} d x^{\nu}-r_{c}^{2} d y^{2}, \quad y \in[0, \pi]
$$

with $x^{\mu}(\mu \in 0,1,2,3)$ coordinates of the usual $4 \mathrm{~d}$ spacetime and $y$ the coordinate of the fourth spatial direction. The quantities $k, r_{c}^{-1}$ are $\mathcal{O}\left(M_{\mathrm{Pl}}\right)$ with $k r_{c} \approx 12$. The "little hierarchy" between $k$ and $r_{c}^{-1}$ can be generated and stabilized naturally [127]. The hierarchy between electroweak and Planck scale is a consequence of the "warped" metric: mass parameters in the fundamental $5 \mathrm{~d}$ model $m_{0}$ appear in our visible space as

$$
m=m_{0} e^{-k r_{c} \pi} \approx m_{0} 10^{-16} .
$$

Hence, all mass scales of the model can be assumed to be of the order of the Planck scale. Many modifications to the original RS Model, concerning which fields should be localised on the branes, have been considered necessary in order to meet electroweak precision and flavour constraints [128]. However, the Higgs field needs to be localised on or near the IR brane in order to explain the hierarchy problem. Therefore, the RS scalar sector is a rather robust prediction of RS models.

\subsubsection{The Higgs-radion system}

Fluctuations of the compactification "radius" $r_{c}$ correspond to a scalar degree of freedom in addition to the $4 \mathrm{~d}$ metric fluctuations. As a consequence of stabilising $r_{c}$, this scalar acquires a vacuum expectation value $(\mathrm{VEV}) \Lambda_{\phi}$ and appears as a massive 
scalar (the radion $\left.\phi_{0}(x)\right)$ in the spectrum $[127,129]$. Higgs-radion mixing may occur via the curvature-scalar interaction [130]

$$
\mathcal{L}=-\xi \sqrt{-g_{\text {ind }}} R\left(g_{\text {ind }}\right) \Phi^{\dagger} \Phi
$$

where $g_{\text {ind }}\left(\phi_{0}(x), \cdots\right)$ is the induced $4 \mathrm{~d}$ metric on the IR brane, $R$ the Ricci scalar, and $\Phi$ the Higgs doublet. Hence, in general, what would be for $\xi=0$ the physical Higgs degree of freedom $h_{0}$ and the radion $\phi_{0}$ mix to form two mass eigenstates $h$ and $\phi$. One arrives at the fields $h_{0}$ and $\phi_{0}$ after rescaling to canonical normalisation on the IR brane. The part of the Lagrangian bilinear in $h_{0}$ and $\phi_{0}$ reads $[131,132]$ :

$$
\mathcal{L}=-\frac{1}{2}\left(1+6 \gamma^{2} \xi\right) \phi_{0} \square \phi_{0}-\frac{1}{2} h_{0} \square h_{0}-6 \gamma \xi \phi_{0} \square h_{0}-\frac{1}{2} m_{\phi_{0}}^{2} \phi_{0}^{2}-\frac{1}{2} m_{h_{0}}^{2} h_{0}^{2} .
$$

Diagonalisation and canonical normalisation of the bilinear terms can be obtained for the physical fields $h$ and $\phi$ via the identification:

$$
\phi_{0}=a \phi+b h, \quad h_{0}=d h+c \phi
$$

with

$$
a=-\frac{\cos \theta}{Z}, \quad b=\frac{\sin \theta}{Z}, \quad c=\sin \theta+\frac{6 \xi \gamma}{Z} \cos \theta, \quad d=\cos \theta-\frac{6 \xi \gamma}{Z} \sin \theta,
$$

and

$$
\tan 2 \theta=\frac{12 \gamma \xi Z m_{h_{0}}^{2}}{m_{\phi_{0}}^{2}-m_{h_{0}}^{2}\left(Z^{2}-36 \xi^{2} \gamma^{2}\right)}, \quad Z^{2}=1+6 \xi \gamma^{2}(1-6 \xi), \quad \gamma=\frac{v}{\Lambda_{\phi}} .
$$

In this formulae, $v(\approx 246 \mathrm{GeV})$ is the Higgs $\mathrm{VEV}$. The radion $\mathrm{VEV} \Lambda_{\phi}$ is a free parameter of the model. The masses and couplings to known matter and gauge bosons of the Higgs-radion sector are determined by choosing values for the four parameters $\Lambda_{\phi}$, the curvature-Higgs coupling parameter $\xi$, and the masses $m_{h}$ and $m_{\phi}$ of the physical fields. However, those parameter choices are subject to theoretical constraints, which come from requiring positive kinetic energy and mass terms in the Lagrangian $[131,132]$ :

$$
Z^{2}>0, \quad \max \left[\frac{m_{h}^{2}}{m_{\phi}^{2}}, \frac{m_{\phi}^{2}}{m_{h}^{2}}\right]>1+\frac{2}{x}(\sqrt{1-x}+1-x),
$$

with $x=\left(1+36 \xi^{2} \gamma^{2} / Z^{2}\right)^{-1}$. The Lagrangian parameters $m_{h^{0}}^{2}$ and $m_{\phi^{0}}^{2}$ are determined for a given valid parameter scenario by

$$
\begin{aligned}
& m_{h^{0}}^{2}=\frac{x}{2}(\Sigma+[-] \Delta), m_{\phi^{0}}^{2}=\frac{Z^{2}}{2}(\Sigma-[+] \Delta), \text { if } m_{h}>m_{\phi}\left[m_{h}<m_{\phi}\right], \\
& \text { with } \Sigma=m_{h}^{2}+m_{\phi}^{2}, \Delta=\sqrt{\Sigma^{2}-\frac{4}{x} m_{h}^{2} m_{\phi}^{2}}
\end{aligned}
$$


In the following, we do not consider scenarios where the physical scalars can decay into Kaluza-Klein (KK) excitations of the graviton, i.e. implicitly we assume that the mass $m_{1}$ of this excitation is large enough to forbid such decay channels. This puts a lower bound on the curvature parameter $k$.

Providing values for $\Lambda_{\phi}, \xi, m_{h}$ and $m_{\phi}$ for a valid scenario, the quantities $a, b, c$, and $d$ defined in Eq. (38) are determined. This, in turn, determines the effective couplings of the two physical scalars $h$ and $\phi$ to gauge bosons and matter normalised to the couplings of a SM Higgs boson with the same mass [120,132]:

$$
\begin{aligned}
& \frac{\Gamma(h \rightarrow f \bar{f})}{\Gamma\left(H_{\mathrm{SM}} \rightarrow f \bar{f}\right)}=\frac{\Gamma\left(h \rightarrow W^{+} W^{-}\right)}{\Gamma\left(H_{\mathrm{SM}} \rightarrow W^{+} W^{-}\right)}=\frac{\Gamma(h \rightarrow Z Z)}{\Gamma\left(H_{\mathrm{SM}} \rightarrow Z Z\right)}=\left|d+\frac{v}{\Lambda_{\phi}} b\right|^{2}, \\
& \frac{\Gamma(\phi \rightarrow f \bar{f})}{\Gamma\left(H_{\mathrm{SM}} \rightarrow f \bar{f}\right)}=\frac{\Gamma\left(\phi \rightarrow W^{+} W^{-}\right)}{\Gamma\left(H_{\mathrm{SM}} \rightarrow W^{+} W^{-}\right)}=\frac{\Gamma(\phi \rightarrow Z Z)}{\Gamma\left(H_{\mathrm{SM}} \rightarrow Z Z\right)}=\left|c+\frac{v}{\Lambda_{\phi}} a\right|^{2}, \\
& \frac{\Gamma(h \rightarrow g g)}{\Gamma\left(H_{\mathrm{SM}} \rightarrow g g\right)}=\frac{v^{2}}{\Lambda_{\phi}^{2}} \frac{\left|2 b b_{3}-\left(b+\frac{\Lambda_{\phi}}{v} d\right) F_{1 / 2}\left(\tau_{t}^{h}\right)\right|^{2}}{\left|F_{1 / 2}\left(\tau_{t}^{h}\right)\right|^{2}}, \\
& \frac{\Gamma(\phi \rightarrow g g)}{\Gamma\left(H_{\mathrm{SM}} \rightarrow g g\right)}=\frac{v^{2}}{\Lambda_{\phi}^{2}} \frac{\left|2 a b_{3}-\left(a+\frac{\Lambda_{\phi}}{v} c\right) F_{1 / 2}\left(\tau_{t}^{\phi}\right)\right|^{2}}{\left|F_{1 / 2}\left(\tau_{t}^{\phi}\right)\right|^{2}}, \\
& \frac{\Gamma^{2}(h \rightarrow \gamma \gamma)}{\Gamma\left(H_{\mathrm{SM}} \rightarrow \gamma \gamma\right)}=\frac{v^{2}}{\Lambda_{\phi}^{2}} \frac{\left|3 b\left(b_{2}+b_{Y}\right)-\left(b+\frac{\Lambda_{\phi}}{v} d\right)\left(4 F_{1 / 2}\left(\tau_{t}^{h}\right)+3 F_{1}\left(\tau_{W}^{h}\right)\right)\right|^{2}}{\left|4 F_{1 / 2}\left(\tau_{t}^{h}\right)+3 F_{1}\left(\tau_{W}^{h}\right)\right|^{2}}, \\
& \frac{\Gamma(\phi \rightarrow \gamma \gamma)}{\Gamma\left(H_{\mathrm{SM}} \rightarrow \gamma \gamma\right)}=\frac{v^{2}}{\Lambda_{\phi}^{2}} \frac{\left|3 a\left(b_{2}+b_{Y}\right)-\left(a+\frac{\Lambda_{\phi}}{v} c\right)\left(4 F_{1 / 2}\left(\tau_{t}^{\phi}\right)+3 F_{1}\left(\tau_{W}^{\phi}\right)\right)\right|^{2}}{\left|4 F_{1 / 2}\left(\tau_{t}^{\phi}\right)+3 F_{1}\left(\tau_{W}^{\phi}\right)\right|^{2}},
\end{aligned}
$$

with $b_{2}=19 / 6, b_{3}=7, b_{Y}=-41 / 6, \tau_{t}^{h[\phi]}=4 m_{t}^{2} / m_{h[\phi]}^{2}, \tau_{W}^{h[\phi]}=4 m_{W}^{2} / m_{h[\phi]}^{2}$, and

$$
\begin{aligned}
F_{1 / 2}(\tau)=-2 \tau(1+(1-\tau) f(\tau)), \quad F_{1}(\tau) & =2+3 \tau+3 \tau(2-\tau) f(\tau), \\
f(\tau) & =\left\{\begin{array}{ll}
\arcsin ^{2}\left(\tau^{-1 / 2}\right), & \tau \geq 1 \\
-\frac{1}{4}\left[\ln \left(\frac{1+\sqrt{1-\tau}}{1-\sqrt{1-\tau}}-i \pi\right)\right] & \tau>1
\end{array} .\right.
\end{aligned}
$$

Like for the Higgs, the radion couplings to massive fermions and gauge bosons are proportional to mass, but e.g. the couplings $\phi_{0} b \bar{b}$ and $\phi_{0} \gamma \gamma$ are suppressed while $\phi_{0} g g$ is enhanced with respect to the SM Higgs boson. In order to consider all possible search channels for the Randall-Sundrum model with HiggsBounds, knowledge of the branching ratios for the decays $h \rightarrow \phi \phi$ and $\phi \rightarrow h h$ is needed as well ${ }^{10}$. However, those channels turn out not to be the most significant ones in the examples we study below.

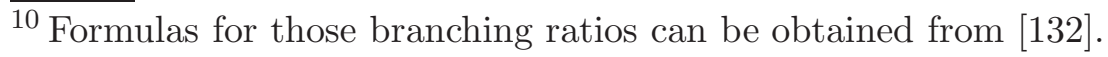




\subsubsection{Constraints}

We chose here a rather extreme scenario with $\Lambda_{\phi}$ as low as $1 \mathrm{TeV}$, the phenomenology of which has been pioneered in [132]. This scenario may already have been excluded by other observations. In particular, our assumption that $m_{1}$ is high enough such that decays of $h$ or $\phi$ into graviton KK excitations are impossible, entails a rather high curvature scale $k$ in conflict e.g. with direct searches for graviton KK excitations in the Randall-Sundrum model [133]. However, it serves demonstration purpose well. To our knowledge, this is the first time that Tevatron Higgs search results have been recast in order to provide constraints on the scalar sector of the Randall-Sundrum model. A more thorough phenomenological study will appear elsewhere [134].

Fig. 4 shows for the RS model the excluded region (left panel) and the search channel with highest sensitivity (right panel) in the $m_{h}-m_{\phi}$ plane, while the two other free parameters in this model are set to $\Lambda_{\phi}=1 \mathrm{TeV}$ and $\xi=1 / 6$. Note the region of parameter space inaccessible due to the theoretical constraints in Eq. (41) shown in Fig. 4 (left panel). This scenario shows slight Higgs-radion mixing, i.e. $h$ behaves mainly like the SM Higgs and $\phi$ mainly like the unmixed radion. This is reflected by the LEP exclusion of $m_{h}$ values up to almost the SM mass limit via the process $e^{+} e^{-} \rightarrow h Z, h \rightarrow b \bar{b}$ (see Fig. 4, right panel) almost independent of $m_{\phi}$. For larger values of $m_{h}$, there is a LEP excluded region via $e^{+} e^{-} \rightarrow \phi Z, \phi \rightarrow$ hadrons for $m_{\phi}$ below about $50 \mathrm{GeV}$. In this region, the enhanced $g g$ coupling and the suppressed $b \bar{b}$ coupling of the radion-like $\phi$ renders this channel the most sensitive one. The exclusion of parameter regions by Tevatron searches is mainly due to the model independent cross section limit on the process $p \bar{p} \rightarrow \operatorname{single} S, S \rightarrow W^{+} W^{-} \rightarrow l \nu l \nu$ [5] for a scalar $S$. Indeed, for scalar masses $m_{h}$ and $m_{\phi}$ above around $120 \mathrm{GeV}$ in the displayed parameter region, this analysis has the highest statistical sensitivity (see Fig. 4, right panel). Furthermore, the highest exclusion power of this analysis is for scalar masses around $2 m_{W} \approx 165 \mathrm{GeV}$. The right panel of Fig. 4 reveals that sizable regions where either $m_{h}$ or $m_{\phi}$ have values close to $165 \mathrm{GeV}$ are excluded by this analysis with $S=h$ or $S=\phi$. For instance for $m_{h}=300 \mathrm{GeV}$, the excluded range of $m_{\phi}$ values due to non-observation of the radion-like scalar $\phi$ is wider than for a SM-like Higgs because of the net enhancement of the signal cross section $\sigma(p \bar{p} \rightarrow g g \rightarrow \phi) \times \mathrm{BR}\left(\phi \rightarrow W^{+} W^{-}\right)$ with respect to the SM.

Fig. 5 shows the excluded Randall-Sundrum parameter space also for $\Lambda_{\phi}=1 \mathrm{TeV}$ but as function of $\xi$ and $m_{\phi}$ with fixed $m_{h}=120 \mathrm{GeV}$. ${ }^{11}$ The parameter exclusion for $m_{\phi}$ above $120 \mathrm{GeV}$ is entirely due to the application of the Tevatron analysis [5] to the radion-like $\phi$, i.e. due to the search channel $p \bar{p} \rightarrow$ single $\phi, \phi \rightarrow W^{+} W^{-} \rightarrow l \nu l \nu$ (see Fig. 5, right panel). In this region, the effective $g g \phi$ coupling is rising with falling $\xi$, while the effective $W W \phi$ coupling goes through a minimum located near $\xi \approx 0$. This explains, on the one hand, the general trend of an increasing excluded $m_{\phi}$-interval with falling $\xi$ values and, on the other hand, the unexcluded funnel region near $\xi \approx 0$.

\footnotetext{
$\overline{{ }^{11} \text { Fig. } 5}$ can be seen as an update of Fig. 18 in [132].
} 

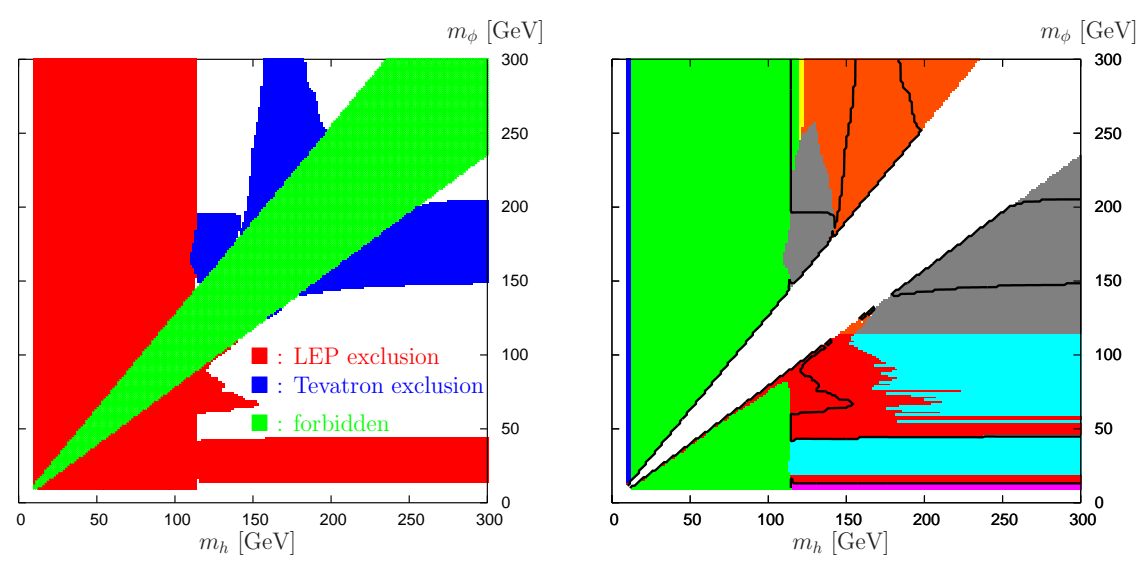

Randall-Sundrum Model (RS1)

$\Lambda_{\phi}=1 \mathrm{TeV}, \xi=1 / 6$

most sensitive analysis:

ㅁ $e^{+} e^{-} \rightarrow h Z, h \rightarrow b \bar{b}$

ㅁ: $e^{+} e^{-} \rightarrow \phi Z, \phi \rightarrow b \bar{b}$

口: $e^{+} e^{-} \rightarrow h Z, h \rightarrow$ anything

n: $e^{+} e^{-} \rightarrow \phi Z, \phi \rightarrow$ anything

: $e^{+} e^{-} \rightarrow \phi Z, \phi \rightarrow$ hadrons

그 $p \bar{p} \rightarrow W h, h \rightarrow b \bar{b}$

ㅁ: $p \bar{p} \rightarrow$ single $h, h \rightarrow W^{+} W^{-}$

ㅁ: $p \bar{p} \rightarrow$ single $\phi, \phi \rightarrow W^{+} W^{-}$

Fig. 4. Excluded region in the $m_{h}-m_{\phi}$ plane of the scalar sector of the original RandallSundrum model (RS1) for $\Lambda_{\phi}=1 \mathrm{TeV}$ and $\xi=1 / 6$ (left panel) and most sensitive search channel (right panel) using HiggsBounds 2.0.0.
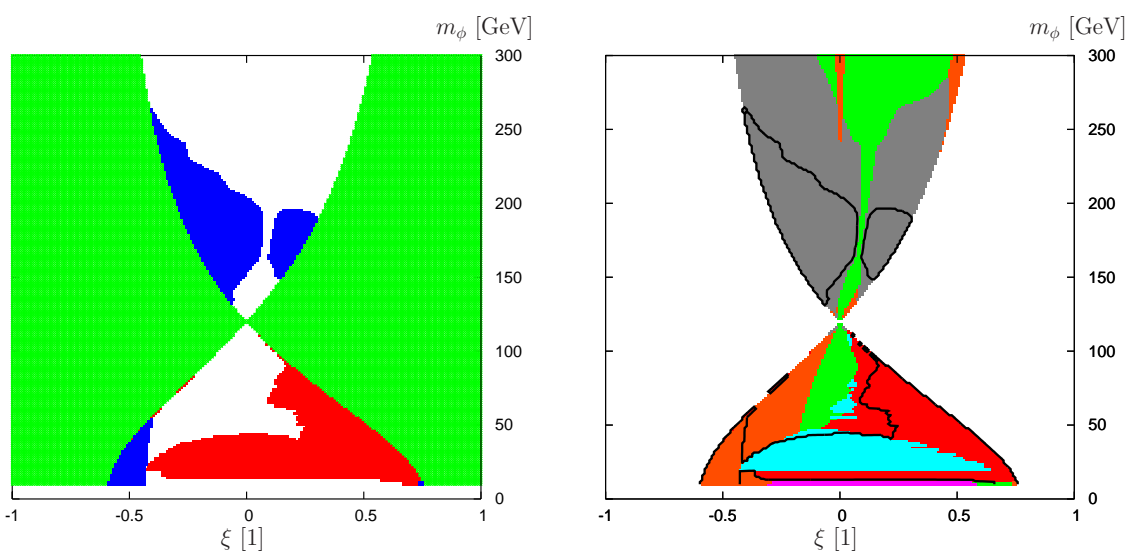

Randall-Sundrum Model (RS1) $\Lambda_{\phi}=1 \mathrm{TeV}, m_{h}=120 \mathrm{GeV}$

most sensitive analysis:

घ: $e^{+} e^{-} \rightarrow h Z, h \rightarrow b \vec{b}$

口: $e^{+} e^{-} \rightarrow \phi Z, \phi \rightarrow b \bar{b}$

口: $e^{+} e^{-} \rightarrow \phi Z, \phi \rightarrow$ anything

ㅍ: $e^{+} e^{-} \rightarrow \phi Z, \phi \rightarrow$ hadrons

घ $: p \bar{p} \rightarrow$ single $h, h \rightarrow W^{+} W^{-}$

च $: p \bar{p} \rightarrow$ single $\phi, \phi \rightarrow W^{+} W^{-}$

Fig. 5. Excluded region in the $\xi-m_{\phi}$ plane of the scalar sector of the original Randall-Sundrum model (RS1) for $\Lambda_{\phi}=1 \mathrm{TeV}$ and $m_{h}=120 \mathrm{GeV}$ (left panel, see Fig. 4 (left panel) for the key) and most sensitive search channel (right panel) using HiggsBounds 2.0.0.

For $m_{\phi}$ below $120 \mathrm{GeV}$, another region is excluded by applying the Tevatron analysis [5] — this time to the Higgs-like $h$. This analysis application becomes the most sensitive one because in the lower left part of the allowed parameter space, both the effective $g g h$ and $W W h$ coupling rise sufficiently with falling $\xi$. The LEP excluded region consists of one region where the most sensitive channel is $e^{+} e-\rightarrow \phi Z, \phi \rightarrow$ hadrons [35-38] (for $m_{\phi}$ roughly below $50 \mathrm{GeV}$ in Fig. 5, right panel), and another one where it is $e^{+} e-\rightarrow \phi Z, \phi \rightarrow b \bar{b}[2]$.

\section{Summary}

We have presented the code HiggsBounds 2.0.0 which allows to test neutral and charged Higgs sectors of arbitrary models against the current exclusion bounds from the Higgs searches at LEP and the Tevatron. The model predictions which are re- 
quired as input to the program, and in particular the three variants of how this information can be provided by the user, are described in detail. Version 2.0.0 represents a significant upgrade of the code since its first release. As a major extension, the code allows now the predictions for (singly) charged Higgs bosons to be confronted with LEP and Tevatron searches. Furthermore, the newly included analyses contain LEP searches for neutral Higgs bosons $(H)$ decaying invisibly or into (non flavour tagged) hadrons as well as decay-mode independent searches, LEP searches via the production modes $\tau^{+} \tau^{-} H$ and $b \bar{b} H$, and Tevatron searches via $t \bar{t} H$. Many updated Tevatron analyses have replaced previously included ones. In particular, all Tevatron results presented at the ICHEP'10 are included in HiggsBounds 2.0.0. We presented a full list of the analyses (with references) incorporated in the program and the implemented conditions for Higgs bosons to be met in order to be applicable to some of the analyses. We provide the complete operating instructions for HiggsBounds2.0.0 with examples, indicating new or extended features with respect to version 1.0.0 of the code.

We have furthermore presented two new physics applications of the program. One application considers a SM-like Higgs boson where an extra Higgs decay channel to invisible particles is open. We find, for instance, that the current Tevatron searches for a SM-like Higgs boson yield an exclusion interval around $m_{H} \approx 2 m_{W}$ even for the case of a Higgs boson which decays invisibly with up to $32 \%$ branching ratio. As a further application we have investigated the scalar sector of the Randall-Sundrum model. Our results show, to our knowledge, for the first time the impact of Tevatron Higgs search results on this model. For the parameter scenario we study here, we find that once the Tevatron results are taken into account in addition to the LEP constraints that had been considered before in [132], large portions of the parameter space are excluded as a consequence of the model-independent Tevatron Higgs search via the decay $H \rightarrow W^{+} W^{-}[5]$.

The functionality of HiggsBounds 2.0.0 is such that upcoming Higgs search limits from the LHC can easily be incorporated. The bounds from Higgs searches at LEP, the Tevatron and the LHC itself will provide an important source of information for testing model interpretations of possible Higgs-like signals, which will hopefully soon emerge from the LHC Higgs searches.

\section{Acknowledgements}

We would like to thank Ralf Bernhard, Peter Bock, Gabriella Pasztor and Yvonne Peters for helpful conversations and input. The work of P.B. was supported by the Helmholtz Young Investigator Grant VH-NG-303. The work of P.B. and G.W. was supported by the Collaborative Research Center SFB676 of the DFG. The work of O.Br. and K.E.W was supported by the Helmholtz Alliance HA-101 'Physics at the Terascale,' The work of S.H. was partially supported by CICYT (grant FPA 
2007-66387 and FPA 2010-22163-C02-01). This work has been supported in part by the European Community's Marie-Curie Research Training Network under contract MRTN-CT-2006-035505 'Tools and Precision Calculations for Physics Discoveries at Colliders' (HEPTOOLS) and under contract MRTN-CT-2006-035657 'Understanding the Electroweak Symmetry Breaking and the Origin of Mass using the First Data of ATLAS' (ARTEMIS). We would like to thank the HepForge development environment, where the web version of HiggsBounds is currently hosted.

\section{References}

[1] LEP Working Group for Higgs boson searches, R. Barate et al., Phys. Lett. B565 (2003) 61, hep-ex/0306033.

[2] ALEPH Collaboration, S. Schael et al., Eur. Phys. J. C47 (2006) 547, hep-ex/0602042.

[3] P. Bechtle et al., Comput. Phys. Commun. 181 (2010) 138, arXiv:0811.4169.

[4] OPAL Collaboration, G. Abbiendi et al., Eur. Phys. J. C27 (2003) 311, hep-ex/0206022.

[5] CDF and D0 Collaborations, T. Aaltonen et al., (2010), arXiv:1005.3216.

[6] D0 Collaboration, Public Note 5739.

[7] D0 Collaboration, V.M. Abazov et al., Phys. Lett. B671 (2009) 349, arXiv:0806.0611.

[8] S. Heinemeyer, W. Hollik and G. Weiglein, Comput. Phys. Commun. 124 (2000) 76, hep-ph/9812320.

[9] S. Heinemeyer, W. Hollik and G. Weiglein, Eur. Phys. J. C9 (1999) 343, hep-ph/9812472.

[10] G. Degrassi et al., Eur. Phys. J. C28 (2003) 133, hep-ph/0212020.

[11] M. Frank et al., JHEP 02 (2007) 047, hep-ph/0611326.

[12] J.S. Lee et al., Comput. Phys. Commun. 156 (2004) 283, hep-ph/0307377.

[13] J.S. Lee et al., Comput. Phys. Commun. 180 (2009) 312, arXiv:0712. 2360.

[14] P. Gondolo et al., JCAP 0407 (2004) 008, astro-ph/0406204.

[15] P. Bechtle, K. Desch and P. Wienemann, Comput. Phys. Commun. 174 (2006) 47, hep-ph/0412012.

[16] O. Buchmueller et al., Phys. Lett. B657 (2007) 87, arXiv:0707.3447.

[17] O. Buchmueller et al., JHEP 09 (2008) 117, arXiv:0808.4128. 
[18] D. Eriksson, J. Rathsman and O. Stal, Comput. Phys. Commun. 181 (2010) 189, arXiv:0902.0851.

[19] A. Djouadi, J. Kalinowski and M. Spira, Comput. Phys. Commun. 108 (1998) 56, hep-ph/9704448.

[20] U. Aglietti et al., (2006), hep-ph/0612172.

[21] T. Figy, C. Oleari and D. Zeppenfeld, Phys. Rev. D68 (2003) 073005, hep-ph/0306109.

[22] O. Brein and W. Hollik, Phys. Rev. D68 (2003) 095006, hep-ph/0305321.

[23] O. Brein and W. Hollik, Phys. Rev. D76 (2007) 035002, arXiv:0705.2744.

[24] O. Brein, Phys. Rev. D81 (2010) 093006, arXiv:1003.4438.

[25] ALEPH Collaboration, Phys. Rept. 427 (2006) 257, hep-ex/0509008.

[26] ALEPH Collaboration, J. Alcaraz et al., (2006), hep-ex/0612034.

[27] A. Djouadi, Phys. Rept. 457 (2008) 1, hep-ph/0503172.

[28] LEP Higgs Working for Higgs boson searches, (2001), hep-ex/0107032.

[29] DELPHI Collaboration, J. Abdallah et al., Eur. Phys. J. C32 (2004) 475, hep-ex/0401022.

[30] L3 Collaboration, P. Achard et al., Phys. Lett. B609 (2005) 35, hep-ex/0501033.

[31] OPAL Collaboration, G. Abbiendi et al., Phys. Lett. B682 (2010) 381, arXiv:0707.0373.

[32] LHWG, LHWG Notes 2002-02.

[33] DELPHI Collaboration, J. Abdallah et al., Eur. Phys. J. C38 (2004) 1, hep-ex/0410017.

[34] OPAL Collaboration, G. Abbiendi et al., Eur. Phys. J. C23 (2002) 397, hep-ex/0111010.

[35] DELPHI Collaboration, J. Abdallah et al., Eur. Phys. J. C44 (2005) 147, hep-ex/0510022.

[36] ALEPH Collaboration, A. Heister et al., Phys. Lett. B544 (2002) 25, hep-ex/0205055.

[37] OPAL Collaboration, G. Abbiendi et al., Phys. Lett. B597 (2004) 11, hep-ex/0312042.

[38] OPAL Collaboration, G. Abbiendi et al., Eur. Phys. J. C40 (2005) 317, hep-ex/0408097.

[39] CDF Collaboration, Public Note 10235. 
[40] CDF Collaboration, T. Aaltonen et al., Phys. Rev. D80 (2009) 071101, arXiv:0908.3534.

[41] D0 Collaboration, Public Note 6089.

[42] CDF Collaboration, T. Aaltonen et al., Phys. Rev. Lett. 102 (2009) 021802, arXiv:0809.3930.

[43] D0 Collaboration, Public Note 6092.

[44] D0 Collaboration, V.M. Abazov et al., Phys. Rev. Lett. 102 (2009) 051803, arXiv:0808.1970.

[45] CDF Collaboration, Public Note 10217.

[46] CDF Collaboration, T. Aaltonen et al., Phys. Rev. Lett. 103 (2009) 101802, arXiv:0906.5613.

[47] D0 Collaboration, V.M. Abazov et al., Phys. Rev. Lett. 101 (2008) 071804, arXiv:0805.2491.

[48] D0 Collaboration, Public Note 5740.

[49] CDF Collaboration, T. Aaltonen et al., Phys. Rev. Lett. 103 (2009) 201801, arXiv:0906.1014.

[50] CDF and D0 TEVNPH Working Group, (2010), arXiv:1003.3363.

[51] D0 Collaboration, Public Note 5873.

[52] CDF Collaboration, Public Note 7307 (version 3).

[53] CDF Collaboration, Public Note 10105.

[54] D0 Collaboration, Public Note 5726.

[55] D0 Collaboration, V.M. Abazov et al., Phys. Rev. Lett. 101 (2008) 221802, arXiv:0805.3556.

[56] D0 Collaboration, V.M. Abazov et al., Phys. Rev. Lett. 104 (2010) 151801, arXiv:0912.0968.

[57] D0 Collaboration, Public Note 5985.

[58] D0 Collaboration, Public Note 6083.

[59] CDF Collaboration, Public Note 10212.

[60] CDF Collaboration, T. Aaltonen et al., Phys. Rev. Lett. 104 (2010) 141801, arXiv:0911.3935.

[61] D0 Collaboration, Public Note 6087.

[62] D0 Collaboration, V.M. Abazov et al., Phys. Rev. Lett. 104 (2010) 071801, arXiv:0912.5285. 
[63] D0 Collaboration, Public Note 6091.

[64] D0 Collaboration, Public Note 5757.

[65] CDF Collaboration, Public Note 10102.

[66] CDF Collaboration, T. Aaltonen et al., Phys. Rev. Lett. 104 (2010) 061803, arXiv: 1001.4468.

[67] D0 Collaboration, Public Note 5871.

[68] D0 Collaboration, Public Note 6082.

[69] D0 Collaboration, V.M. Abazov et al., Phys. Rev. Lett. 104 (2010) 061804, arXiv: 1001.4481

[70] CDF and D0, T. Aaltonen et al., Phys. Rev. Lett. 104 (2010) 061802, arXiv: 1001.4162.

[71] CDF Collaboration, Public Note 9248.

[72] D0 Collaboration, Public Note 5845.

[73] D0 Collaboration, V.M. Abazov et al., Phys. Rev. Lett. 102 (2009) 251801, arXiv:0903.4800.

[74] CDF Collaboration, Public Note 10010.

[75] D0 Collaboration, Public Note 5858.

[76] D0 Collaboration, V.M. Abazov et al., Phys. Rev. Lett. 102 (2009) 231801, arXiv:0901.1887.

[77] CDF Collaboration, Public Note 10065.

[78] D0 Collaboration, V.M. Abazov et al., Phys. Lett. B663 (2008) 26, arXiv:0712.0598.

[79] CDF and D0 TEVNPH Working Group, (2007), arXiv:0712.2383.

[80] CDF and D0 TEVNPH Working Group, (2008), arXiv:0804.3423.

[81] CDF and D0 TEVNPH Working Group, (2008), arXiv:0808.0534.

[82] CDF Collaboration, Public Note 9999.

[83] D0 Collaboration, Public Note 6008.

[84] CDF Collaboration, (2009), arXiv:0911.3930.

[85] CDF and D0 TEVNPH Working Group, (2010), arXiv:1007.4587.

[86] LEP Higgs Working Group for Higgs boson searches, (2001), hep-ex/0107031.

[87] DELPHI Collaboration, J. Abdallah et al., Eur. Phys. J. C34 (2004) 399, hep-ex/0404012. 
[88] D0 Collaboration, V.M. Abazov et al., Phys. Lett. B682 (2009) 278, arXiv:0908.1811.

[89] CDF Collaboration, T. Aaltonen et al., Phys. Rev. Lett. 103 (2009) 101803, arXiv:0907.1269.

[90] D. Graudenz, M. Spira and P.M. Zerwas, Phys. Rev. Lett. 70 (1993) 1372.

[91] M. Spira et al., Nucl. Phys. B453 (1995) 17, hep-ph/9504378.

[92] R.V. Harlander and W.B. Kilgore, Phys. Rev. Lett. 88 (2002) 201801, hep-ph/0201206.

[93] V. Ravindran, J. Smith and W.L. van Neerven, Nucl. Phys. B665 (2003) 325, hep-ph/0302135.

[94] C. Anastasiou and K. Melnikov, Nucl. Phys. B646 (2002) 220, hep-ph/0207004.

[95] S. Catani et al., JHEP 07 (2003) 028, hep-ph/0306211.

[96] S. Moch and A. Vogt, Phys. Lett. B631 (2005) 48, hep-ph/0508265.

[97] E. Laenen and L. Magnea, Phys. Lett. B632 (2006) 270, hep-ph/0508284.

[98] O. Brein, A. Djouadi and R. Harlander, Phys. Lett. B579 (2004) 149, hep-ph/0307206.

[99] M.L. Ciccolini, S. Dittmaier and M. Kramer, Phys. Rev. D68 (2003) 073003, hep-ph/0306234.

[100] Higgs Working Group, K.A. Assamagan et al., (2004), hep-ph/0406152.

[101] R.V. Harlander and W.B. Kilgore, Phys. Rev. D68 (2003) 013001, hep-ph/0304035.

[102] T. Han, G. Valencia and S. Willenbrock, Phys. Rev. Lett. 69 (1992) 3274, hep-ph/9206246.

[103] J.M. Campbell and R.K. Ellis, Phys. Rev. D60 (1999) 113006, hep-ph/9905386.

[104] E.L. Berger and J.M. Campbell, Phys. Rev. D70 (2004) 073011, hep-ph/0403194.

[105] W. Beenakker et al., Phys. Rev. Lett. 87 (2001) 201805, hep-ph/0107081.

[106] L. Reina and S. Dawson, Phys. Rev. Lett. 87 (2001) 201804, hep-ph/0107101.

[107] S. Dawson et al., Phys. Rev. D67 (2003) 071503, hep-ph/0211438.

[108] M.S. Carena et al., Eur. Phys. J. C26 (2003) 601, hep-ph/0202167.

[109] Particle Data Group, C. Amsler et al., Phys. Lett. B667 (2008) 1.

[110] M. Jezabek and J.H. Kuhn, Nucl. Phys. B314 (1989) 1.

[111] See http://www.feynhiggs.de/.

[112] K. Griest and H.E. Haber, Phys. Rev. D37 (1988) 719. 
[113] A. Djouadi et al., Phys. Lett. B376 (1996) 220, hep-ph/9603368.

[114] T. Binoth and J.J. van der Bij, Z. Phys. C75 (1997) 17, hep-ph/9608245.

[115] F. de Campos et al., Phys. Rev. D55 (1997) 1316, hep-ph/9601269.

[116] Y. Chikashige, R.N. Mohapatra and R.D. Peccei, Phys. Lett. B98 (1981) 265.

[117] A.S. Joshipura and S.D. Rindani, Phys. Rev. Lett. 69 (1992) 3269.

[118] K. Belotsky et al., Phys. Rev. D68 (2003) 054027, hep-ph/0210153.

[119] I. Antoniadis, M. Tuckmantel and F. Zwirner, Nucl. Phys. B707 (2005) 215, hep-ph/0410165.

[120] G.F. Giudice, R. Rattazzi and J.D. Wells, Nucl. Phys. B595 (2001) 250, hep-ph/0002178.

[121] S.P. Martin and J.D. Wells, Phys. Rev. D60 (1999) 035006, hep-ph/9903259.

[122] A. Datta et al., Phys. Rev. D70 (2004) 075003, hep-ph/0404056.

[123] D. Dominici and J.F. Gunion, Phys. Rev. D80 (2009) 115006, arXiv:0902.1512.

[124] I. Antoniadis et al., Phys. Lett. B436 (1998) 257, hep-ph/9804398.

[125] N. Arkani-Hamed, S. Dimopoulos and G.R. Dvali, Phys. Lett. B429 (1998) 263, hep-ph/9803315.

[126] L. Randall and R. Sundrum, Phys. Rev. Lett. 83 (1999) 3370.

[127] W.D. Goldberger and M.B. Wise, Phys. Rev. Lett. 83 (1999) 4922.

[128] S. Casagrande et al., JHEP 10 (2008) 094, arXiv:0807.4937, and references therein.

[129] W.D. Goldberger and M.B. Wise, Phys. Lett. B 475 (2000) 275.

[130] S.L. Adler, Rev. Mod. Phys. 54 (1982) 729, and references therein.

[131] C. Csaki, M.L. Graesser and G.D. Kribs, Phys. Rev. D63 (2001) 065002, hep-th/0008151.

[132] D. Dominici et al., Nucl. Phys. B671 (2003) 243, hep-ph/0206192.

[133] CDF Collaboration, T. Aaltonen, (2010), arXiv:1012.2795.

[134] O. Brein, in preparation. 